(C) 2018 IEEE. Personal use of this material is permitted. Permission from IEEE must be obtained for all other uses, in any current or future media, including reprinting/republishing this material for advertising or

promotional purposes, creating new collective works, for resale or redistribution to servers or lists, or reuse of any copyrighted component of this work in other works. 


\title{
BDPK: Bayesian Dehazing Using Prior Knowledge
}

\author{
Mingye Ju, Student Member, IEEE, Can Ding, Member, IEEE, Dengyin Zhang, Member, IEEE, \\ and Y. Jay Guo, Fellow, IEEE
}

\begin{abstract}
Atmospheric scattering model (ASM) has been widely used in hazy image restoration. However, the recovered albedo might deviate from the real scene once the input hazy image cannot fully satisfy the model's assumptions such as the homogeneous atmosphere and even illumination. In this paper, we break these limitations and redefine a more reliable atmospheric scattering model (RASM) that is extremely adaptable for various practical scenarios. Benefiting from RASM, a simple yet effective Bayesian dehazing algorithm (BDPK) is further proposed based on the prior knowledge. Our strategy is to convert the single image dehazing problem into a maximum a-posteriori probability (MAP) one that can be approximated as an optimization function using the existing priori constraints. To efficiently solve this optimization function, the alternating minimizing technique (AMT) is introduced, which enables us to directly restore the scene albedo. Experiments on a number of challenging images reveal the power of BDPK on removing haze and verify its superiority over several state-of-the-art techniques in terms of quality and efficiency.
\end{abstract}

Index Terms-Image Haze Removal, Atmospheric Scattering Model, Bayesian Theory, Depth Map, Scattering Distribution.

\section{INTRODUCTION}

Under bad weather conditions, the irradiance of objects is absorbed and scattered by the atmospheric suspended particles before reaching the camera. Images captured in inclement weather are often plagued by low visibility and thus result in contrast reduction and color distortion as shown in Fig. 1(a). This poor quality is bound to seriously degenerate the performance of computer vision applications that are primarily designed for high-quality inputs, such as surveillance [1], [2], intelligent vehicles [3] and object recognition [4], [5]. To make the buried information visible, an effective and applicable single image dehazing technique is urgent needs.

It is well-known that image haze removal is an inherently ill-posed issue since measuring scene depth is difficult for cameras. Directly employing traditional image enhancement methods [6]-[14] to restore the contrast of hazy images may be the most intuitive and simplest way to recall the visibility in buried regions. However, these techniques are somehow limited due to the ignored degrading mechanism [15]. For example, histogram equalization (HE) [6] improves the global contrast of the input image by stretching the dynamic range for the color channels, yet it lacks the capability of enlarging the local visibility in each region. Although the adaptive

Manuscript accepted September 3, 2018.

M. Ju and D. Zhang are with the School of Internet of Things, Nanjing University of Posts and Telecommunications, Nanjing, 210000, China. $\mathrm{M}$. $\mathrm{Ju}$ is also with the Global Big Data Technologies Centre (GBDTC), University of Technology Sydney (UTS), Ultimo, NSW, 2007, Australia. email:(2014070245@njupt.edu.cn)

C. Ding and Y. Jay Guo are with the Global Big Data Technologies Centre (GBDTC), University of Technology Sydney (UTS), Ultimo, NSW, 2007, Australia. e-mail:(can.ding.1989@gmail.com)

Corresponding authors: Dengyin Zhang and Can Ding histogram equalization (AHE) [7] effectively overcomes the above defect, the over-enhancement may be aroused and its large computational complexity has to be considered. Retinex methods $[8]-[10]$ have made great progress through decades of development. They realize better dynamic range compression and the tonal rendition. Regrettably, the poor edge-preserving ability would give rise to halo artifacts in discontinuous areas [16]. The core idea of gamma correction [11] and nonlinear stretching operation [12] is to revise individual pixel value without considering its neighbor content, thus their enhanced results lack visual realism. Homogeneous filtering [13] jointly utilizes the frequency filtering and grayscale transformation to resume the target clarity of the input image, but the recovery quality relies on the parameter initialization. Detail enhancement [14] increases the high-frequency details by sharply highlighting the object contours in the whole image. Undoubtedly, it is difficult to reach a better trade-off between the over-sharpening of close-range regions and the weaksharpening of long-range ones.

Benefiting from atmospheric scattering theory [15], [17], Narasimhan et al. [18] proposed the haze removal strategy using two images of the same scene taken in different atmospheric conditions. The premise of using this method is that the original input should contain the infinite far-point and near-point. Otherwise, over-saturation will be introduced into the small-depth areas. In [19], Kopf et al. exploited the given geo-referenced digital terrain and urban models to extract the scene depth, and then automatically conducted the haze removal. According to the polarization characteristics, Schechner et al. [20], [21] utilized different polarization angles from two of same scenes in order to remove the haze from the degraded images. Generally, these early approaches are capable of achieving satisfactory recovery results, but these additional requirements must be hard to fulfill. Thus, their methods lack practicality in many real applications.

Recently, single image dehazing techniques have attracted much research attention. The success of these techniques lies in using the strong priori constraints. For instance, Tan [22] successfully removed the haze cover in an input based on the fact that clear-day image has richer contrast than the corresponding one polluted by haze. This method is very positive for dense hazy images, but the resumed colors for misty scenes are prone to be over-saturation. Fattal [23] assumed that surface shading and medium transmissions are uncorrelated in a local patch, and the haze is eliminated by utilizing independent component analysis (ICA). Obviously, this technique is challenged when the images contain less color information. Later in [24], Fattal further derived a local model that explains the color-lines meaning in vague region and used it to seek the more accurate transmission. 
Still, this approach may fail with the mono-color images where the notion of color-lines is trivialized. The significant contribution of the dark channel prior (DCP) [25] allows us to directly detect the rough haze thickness, thereby recovering the realistic results by refining the initial transmission using soft matting (SM) [26]. Unfortunately, this prior cannot fully suitable to the case where the scene brightness is naturally similar to the airlight. Besides, DCP's results always appear too dark and this approach is time-consuming due to the complex SM. To improve the recovery quality of the DCP, the dynamic repair strategy [27], the $\mathrm{I}_{2}$-norm-based DCP [28], and the Laplacian-based mechanism [29] were proposed to boost the performance of estimated transmission. Fisher's linear discriminant-based scheme [30] was designed to exclude the interference of localized light sources and the scene radiance constraint [31] was provided to tackle the darkness problem of dehazed scenes. For efficiency, Huang ${ }^{1}$ et al. [32] defined a hybrid DCP for circumventing halo effects in the recovered results, Gibson et al. [33], Huang ${ }^{2}$ et al. [34], $\mathrm{He}^{1}$ et al. [14], Yu et al. [35] and Xiao et al. [36] sought the replacements of SM to reduce the computational complexity, such as the median filter, improved median filter [34], guided image filtering [14] and guided joint bilateral filtering [35], [36]. From the geometric perspective of DCP, Meng et al. [37] presented the dehazing algorithm with a boundary constraint and contextual regularization. This method can thoroughly unveil the scene structures of interesting parts, yet it does not fundamentally solve the inherent shortcomings of DCP. The strategy proposed by Chen et al. [38], which includes two modules designed using Bi-Histogram modification, is able to produce the restored results with satisfactory visual quality. Nevertheless, the rebuilt sky would suffer from serious color shift when the constant-airlight assumption is invalid. Relying on the difference structure preservation prior, $\mathrm{He}^{2}$ et al. [39] computed the scene transmission by assuming that each patch can be linearly represented to a dictionary. Lai [40] introduced both theoretic and heuristic bounds to restrict the solution space, and designed two objectives for scene priors to excavate the optimal transmission. Kim et al. [41] estimated the transmission using the defined cost function that consists of the contrast term and the information loss term, and its dehazing effect can be adjusted by manually setting the coefficient involved in the function. Based on the color attenuation prior (CAP), Zhu et al. [42] created a linear model and determined the parameters in this model with a supervised learning method, which makes mining the depth structure task more convenient. Regrettably, the recovery quality is not guaranteed due to the unspecified scattering coefficient.

Taking advantage of the machine learning framework (MLF), Tang et al. [43], Cai et al. [44] and Ren et al. [45] provided a new clue for haze removal by blending the established assumptions/priors to train the Dehaze-Systems. Although their systems can remedy the weaknesses of hazerelevant features to a large extent, the intricate MLF employed is bound to reduce the real-time performance. Galdran et al. proposed the perceptual color correction framework (PCCF) [46] and the enhancement framework named STRESS [47], which performs image dehazing from a single input. To

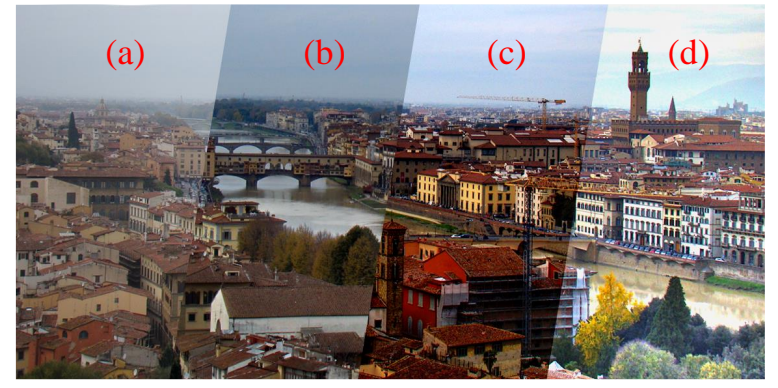

Fig. 1. Comparison with fusion-based dehazing techniques. (a) Hazy region. (b) Ancuti et al.'s result. (c) Choi et al.'s result. (d) BDPK's result

obtain more realistic results, their team modified the previous PCCF and further developed a fusion-based variational imagedehazing (FVID) [48] to maintain high contrast in long-range regions while preserving reasonable content in the close-range ones. Another solution advocated by Nishino and Kratz [49] adopts the Bayesian posterior probability model to remove haze by fully leveraging the latent statistical structures. This approach can have the very promising results for most cases, whereas it may yield some dark artifacts in regions approaching infinite depth. Moreover, the factorial Markov random field (FMRF) employed in this method has much computational overhead, which influences the application prospects of this method.

In this paper, based on our previous work [50] and the fact that inhomogeneous atmosphere phenomenon does exist in the real world [42], we redefine the imaging parameters in the atmospheric scattering model (ASM) in order to improve the model's universality. Benefitting from this redefined model (RASM) and inspired by [49], a simple but effective single image dehazing algorithm that uses Bayesian theory is further proposed. More concretely, the haze removal optimization function in terms of the scattering distribution map, depth map and scene albedo is first deduced by relying on the existing prior knowledge. Then, by combining the optimization function and the introduced alternating minimizing technique (AMT), we can recover the visually realistic result and obtain two by-products of haze removal (scene depth and scattering distribution map). The experimental results, as shown in Fig. 1, demonstrate that the proposed BDPK achieves better restoration of the edge contrast and color vividness compared with the famous fusion-based dehazing techniques including Ancuti et al. [51] and Choi et al. [52].

\section{Refined Atmospheric ScAttering Model}

In machine vision and computer graphics, the atmospheric scattering model (ASM) [15], [17], [18] is widely used to describe the formation of hazy images (see Figs. 2(a,b)). This model can be simply expressed as

$$
\boldsymbol{I}(x, y)=\boldsymbol{A} \cdot \boldsymbol{\rho}(x, y) \cdot t(x, y)+\boldsymbol{A} \cdot(1-t(x, y))
$$

where $\boldsymbol{I}$ is a hazy image, $\boldsymbol{\rho}$ is the expected scene albedo, $\boldsymbol{A}$ is the atmospheric light that is usually assumed as a constant [22]-[25], [27]-[45], [49], and $t$ is the medium transmission. In Model (1), the first term on the right-hand side is called 


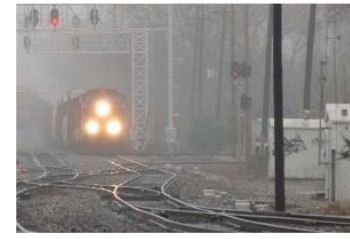

(a)

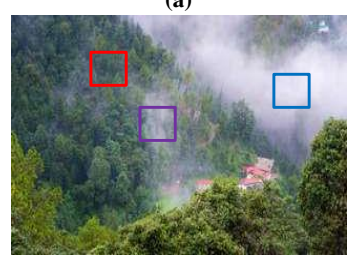

(c)

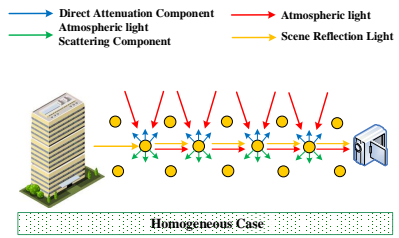

(b)

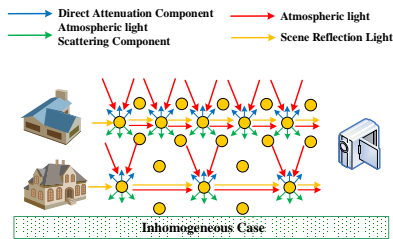

(d)
Fig. 2. (a) Homogeneous atmosphere case. (b) The atmosphere scattering model illustrated for homogeneous case. (c) Inhomogeneous atmosphere case (The distribution of suspended particles varies among the different color boxes). (d) The atmosphere scattering model illustrated for inhomogeneous case.

Direct Attenuation, and it describes the direct impact of scene's reflected light from suspended particles. The second term is Airlight which demonstrates the color shift caused by the scattered light. When the atmospheric distribution is homogeneous, the transmission $t$ is defined as

$$
t(x, y)=e^{-\beta_{0} \cdot d(x, y)}
$$

where $\beta_{0}$ and $d$ are the scattering coefficient and the scene depth, respectively. Equations (1) (2) indicate that the scene albedo is exponentially attenuated with scene depth. Our preliminary work [50] has unveiled that the traditional ASM is invalid for the uneven illumination condition, and this defect can be addressed by redefining the global atmospheric light $\boldsymbol{A}$ as the scene incident light $\boldsymbol{S}$ (More technical details can be found in our study [50]).

In this paper, we further consider the spatially inhomogeneous phenomenon of particle distributions that exists in some specific scenarios. This means that $\beta_{0}$-constant assumption would face the difficulties when processing the inhomogeneous cases (see Figs. 2(c,d)). Therefore, we intend to break this plausible assumption, that is, redefine the constant $\beta_{0}$ as the scattering distribution $\beta$ that varies along with the pixel location. Based on above analysis, a more reliable atmospheric scattering model (RASM) can be described as

$$
\begin{aligned}
\boldsymbol{I}(x, y)= & \boldsymbol{S}(i) \cdot \boldsymbol{\rho}(x, y) \cdot e^{-\beta(x, y) \cdot d(x, y)} \\
& +\boldsymbol{S}(i) \cdot\left(1-e^{-\beta(x, y) \cdot d(x, y)}\right)
\end{aligned}
$$

where $\boldsymbol{S}(i)$ is the incident light of the $i$ th scene [50]. Although RASM is more in line with the real world compared to ASM, it is a more challenge task when using the proposed model to remove the haze because the available structure information is significantly insufficient.

\section{PRoposed BDPK}

As we explained in Section I, the success of single image haze removal depends on the validity of the prior knowledge, whereas the limitations of the aforementioned priors are inevitable in some exceptional circumstances. To this end, we develop a fast image dehazing technique called BDPK based on the Bayesian theory and the RASM. It can compensate for these shortcomings by fully leveraging the latent relationships of the image priors, and thereby recover the more visually comfortable results.

\section{A. MAP Model}

Motivated by [49], [53]-[55], the key step of BDPK is to factorize the hazy image $I$ into the scene albedo $\rho$, the scene depth $d$ and the scattering distribution $\beta$ for each pixel across the entire input. To avoid (3) being nonnegative, we first reverse it as

$$
\boldsymbol{S}(i)-\boldsymbol{I}(x, y)=\boldsymbol{S}(i) \cdot(1-\boldsymbol{\rho}(x, y)) \cdot e^{-\beta(x, y) \cdot d(x, y)}
$$

Afterwards, taking the logarithm operation of each side in (4) yields

$$
\begin{aligned}
\ln (\boldsymbol{S}(i)-\boldsymbol{I}(x, y))= & \ln (\boldsymbol{S}(i) \cdot(1-\boldsymbol{\rho}(x, y))) \\
& -\beta(x, y) \cdot d(x, y)
\end{aligned}
$$

Considering the noise from sensors is another important degradation factor [54] and letting $\boldsymbol{I}^{\prime}(x, y)=\ln (\boldsymbol{S}(i)-\boldsymbol{I}(x, y))$, $\boldsymbol{\rho}^{\prime}(x, y)=\ln (\overrightarrow{\boldsymbol{S}}(i)-\boldsymbol{S}(i) \cdot \boldsymbol{\rho}(x, y))$, Equation (5) is simplified as

$$
\boldsymbol{I}^{\prime}(x, y)=\boldsymbol{\rho}^{\prime}(x, y)-\beta(x, y) \cdot d(x, y)+n(x, y)
$$

where $n$ denotes additive noise. If the incident light $\boldsymbol{S}$ has been estimated using [50], we can regard $\boldsymbol{I}^{\prime}$ as the "new hazy image" since it is a known component and contains all the information of $\boldsymbol{I}$. Similarly, $\boldsymbol{\rho}^{\prime}$ can be considered as the "hazefree image".

According to the Bayesian theory, the posterior probability constituted by all parameters in (6) can be described as

$$
p\left(\boldsymbol{\rho}^{\prime}, \beta, d \mid \boldsymbol{I}^{\prime}\right)=\frac{p\left(\boldsymbol{I}^{\prime} \mid \boldsymbol{\rho}^{\prime}, \beta, d\right) \cdot p\left(\boldsymbol{\rho}^{\prime} \mid \beta, d\right) \cdot p(\beta \mid d) \cdot p(d)}{p\left(\boldsymbol{I}^{\prime}\right)}
$$

where $p\left(\boldsymbol{I}^{\prime}\right)$ is a determined constant due to the given $\boldsymbol{I}^{\prime}$. Moreover, it can be noticed that $\boldsymbol{\rho}^{\prime}, \beta$, and $d$ are totally uncorrelated in the real world. Therefore, Equation (7) is further equivalent to

$$
p\left(\boldsymbol{\rho}^{\prime}, \beta, d \mid \boldsymbol{I}^{\prime}\right) \propto p\left(\boldsymbol{I}^{\prime} \mid \boldsymbol{\rho}^{\prime}, \beta, d\right) \cdot p\left(\boldsymbol{\rho}^{\prime}\right) \cdot p(\beta) \cdot p(d)
$$

To restore the "haze-free image" $\rho$ ', we take the logarithm operation of each side in (8) and then maximize its posterior probability. Accordingly, the maximum a-posteriori probability (MAP) model for haze removal can be expressed as

$$
\begin{aligned}
& \operatorname{argmax}\left\{p\left(\boldsymbol{\rho}^{\prime}, \beta, d \mid \boldsymbol{I}^{\prime}\right)\right\} \\
& \propto \operatorname{argmin}\left\{-\ln \left(p\left(\boldsymbol{I}^{\prime} \mid \boldsymbol{\rho}^{\prime}, \beta, d\right)\right)-\right. \\
& \left.\ln \left(p\left(\boldsymbol{\rho}^{\prime}\right)\right)-\ln (p(\beta))-\ln (p(d))\right\}
\end{aligned}
$$

where we have explicitly illustrated the inner relationship among all the probability density functions of imaging factors in (6). This is the strong foundation for approximating the optimization function of image haze removal. 


\section{B. Model Approximation}

The MAP model for haze removal is given, but it is a fundamentally under-constrained problem due to the insufficient available information. Fortunately, some image priors have been explored. This inspires us to design each probability density function (PDF) to approximate an optimization function corresponding to (9), which enables us to directly restore the scene albedo.

Definition I: To alleviate the model's complexity, we assume the additive noise $n$ is white Gaussian noise. Additionally, based on the fact that the relationship among all the parameters is constrained to (6) [Model Constraint Prior], we might as well define

$$
p\left(\boldsymbol{I}^{\prime} \mid \boldsymbol{\rho}^{\prime}, \beta, d\right)=e^{-\frac{\left\|\boldsymbol{I}^{\prime}-\boldsymbol{\rho}^{\prime}+\beta \cdot d\right\|_{2}^{2}}{\lambda_{1}}}
$$

where $\lambda_{1}$ is the variance of the exponential power distribution (Please note that the subsequent parameters $\lambda_{2}, \lambda_{3}$ and $\lambda_{4}$ have the same meaning as $\lambda_{1}$ ). The above PDF restricts the solution space of all unknown components and thus ensures the authenticity of the recovery result.

Definition II: Clear-day images possess higher visibility than the corresponding hazy ones [22], which indicates that richer image contrast information results in a higher probability that the scene is a real scene [Contrast Prior]. Following this hypothesis, the PDF of $\rho^{\prime}$ is defined as

$$
p\left(\boldsymbol{\rho}^{\prime}\right)=e^{-\frac{\left\|\left(F-\nabla \rho^{\prime}\right)\right\|_{2}^{2}}{\lambda_{2}}}
$$

where $F$ is the maximum upper limit of $\nabla \rho^{\prime}$. In addition to the highlighted pure-white objects, we roughly generalize that $\nabla \boldsymbol{\rho}^{\prime} \in[0,4.6]$ due to $\boldsymbol{S}(i) \cdot(1-\boldsymbol{\rho}(x, y)) \in[0.01,1]$ in general. Thus, we set $F=5$ in this work. Although the contrast prior could basically reflect the objective discipline of the scene albedo, the results recovered by Tan [22] indicate that solely stressing the enhanced of visibility in the degraded image may lead to the over-saturated phenomenon. To address the limitation of the contrast prior used in the PDF (11), an esthesia matrix on haze density is introduced. It is expressed as

$$
W=e^{-\frac{(\bar{I}-1)^{2}}{\sigma}} \cdot e^{-\frac{\left(I_{s}\right)^{2}}{\sigma}}
$$

where $\sigma=0.3$ is a prescribed coefficient, $\bar{I}$ and $I_{s}$ are the brightness component and saturation component of input $\boldsymbol{I}$, respectively. It is obvious that the quantization value of the matrix is proportional to scene brightness and inversely proportional to saturation, which conforms to human visual perception (HVP) for natural haze [42]. Taking this esthesia matrix (12), the final PDF of $\rho^{\prime}$ can be rewritten as

$$
p\left(\boldsymbol{\rho}^{\prime}\right)=e^{-W \cdot \frac{\left\|\left(F-\nabla \rho^{\prime}\right)\right\|_{2}^{2}}{\lambda_{2}}}
$$

where $W$ is able to flexibly control the contrast weights for all pixels with respect to the HVP. Generally, a thicker haze density results in a greater contrast weight. This strategy promises the restoration quality for dense hazy scenes and avoids the over-enhancement of the misty ones.

Definition III: A "good" depth map should preserve the overall depth structure consistent with the original hazy image, and with minimal texture detail simultaneously [25], [41] [Structure Prior]. Hence, the PDF of $d$ can be defined as

$$
p(d)=e^{\frac{-\gamma_{1} \cdot\|d-\tilde{d}\|_{2}^{2}-\gamma_{2} \cdot\|\nabla d\|_{2}^{2}-\gamma_{3} \cdot\|\nabla d-\nabla \bar{I}\|_{2}^{2}}{\lambda_{3} \cdot\left(\gamma_{1}+\gamma_{2}+\gamma_{3}\right)}}
$$

where $\gamma_{1}, \gamma_{2}$ and $\gamma_{3}$ are weight coefficients and $\tilde{d}$ represents the initial estimation of the scene depth. Referring to [49], [53], the minimal component of $\boldsymbol{I}$ can be regarded as the closest depth, thus we initialize this component as the initial depth, that is

$$
\tilde{d}=\min _{c \in\{\mathrm{R}, \mathrm{G}, \mathrm{B}\}}\left(I^{c}(x, y)\right)
$$

where $c$ denotes color channel index and $I^{c}$ is a color channel of $\boldsymbol{I}$.

Definition IV: Similar to scene depth, we notice that the scattering distribution equally shares the original spatial structure and local smoothing feature (see Fig. 2(c)). Based on this observation, the PDF of $\beta$ is defined as

$$
p(\beta)=e^{\frac{-\gamma_{4} \cdot\|\beta-\tilde{\beta}\|_{2}^{2}-\gamma_{5} \cdot\|\nabla \beta\|_{2}^{2}-\gamma_{6} \cdot\|\nabla \beta-\nabla \bar{I}\|_{2}^{2}}{\lambda_{4} \cdot\left(\gamma_{4}+\gamma_{5}+\gamma_{6}\right)}}
$$

where $\gamma_{4}, \gamma_{5}$, and $\gamma_{6}$ are weight coefficients and $\tilde{\beta}$ represents the initial estimation of the scattering distribution. Here, two different atmospheric conditions are required to be taken into account: 1) Regarding the homogeneous case, we set $\tilde{\beta}=1$, which has been proven to be ample for most situations [42]; 2) The brightness of pixels in an inhomogeneous image usually sharply varies along with the change of the concentration of atmospheric particles (see Fig. 2(c)). Thus, we initialize the estimation $\tilde{\beta}=\bar{I}$ for inhomogeneous case.

Optimization Function: Substituting the PDFs (10) (13) (14) (16) into the MAP model (9), the optimization function for haze removal can be approximated as

$$
\begin{aligned}
& \operatorname{argmin}\left\{p\left(\boldsymbol{\rho}^{\prime}, \beta, d \mid \boldsymbol{I}^{\prime}\right)\right\} \\
& =\operatorname{argmin}\left\{\theta_{1} \cdot\left\|\boldsymbol{I}^{\prime}-\boldsymbol{\rho}^{\prime}+\beta \cdot d\right\|_{2}^{2}+\theta_{2} \cdot W \cdot\left\|\nabla \boldsymbol{\rho}^{\prime}-F\right\|_{2}^{2}\right. \\
& +\theta_{3} \cdot\|d-\tilde{d}\|_{2}^{2}+\theta_{4} \cdot\|\nabla d\|_{2}^{2}+\theta_{5} \cdot\|\nabla d-\nabla \bar{I}\|_{2}^{2}+\theta_{6} \\
& \left.\cdot\|\beta-\tilde{\beta}\|_{2}^{2}+\theta_{7} \cdot\|\nabla \beta\|_{2}^{2}+\theta_{8} \cdot\|\nabla \beta-\nabla \bar{I}\|_{2}^{2}\right\}
\end{aligned}
$$

where $\theta_{1}=\frac{1}{\lambda_{1}}, \theta_{2}=\frac{1}{\lambda_{2}}, \theta_{3}=\frac{\gamma_{1}}{\lambda_{3} \cdot\left(\gamma_{1}+\gamma_{2}+\gamma_{3}\right)}, \theta_{4}=$ $\frac{\gamma_{2}}{\lambda_{3} \cdot\left(\gamma_{1}+\gamma_{2}+\gamma_{3}\right)}, \theta_{5}=\frac{\lambda_{2}}{\lambda_{3} \cdot\left(\gamma_{1}+\gamma_{2}+\gamma_{3}\right)}, \theta_{6}=\frac{1}{\lambda_{4} \cdot\left(\gamma_{4}+\gamma_{5}+\gamma_{6}\right)}$, $\theta_{7}=\frac{\gamma_{5}}{\lambda_{4} \cdot\left(\gamma_{4}+\gamma_{5}+\gamma_{6}\right)}$ and $\theta_{8}=\frac{\gamma_{6}}{\lambda_{4} \cdot\left(\gamma_{4}+\gamma_{5}+\gamma_{6}\right)}$ are regular parameters. Minimizing function (17) is computationally intractable because of too many unknown components. In the following section, we will introduce an efficient optimization technique to obtain $\boldsymbol{\rho}^{\prime}, \beta$ and $d$.

\section{Effective Resolving with AMT}

Instinctively, we employ the alternating minimizing technique (AMT) [37], [56] to solve the optimization function (17). The core idea of AMT is to alternatively minimize $\beta, d$, and $\rho^{\prime}$ by assuming that the other components are known. This process is repeated until they converge. Specifically, function 


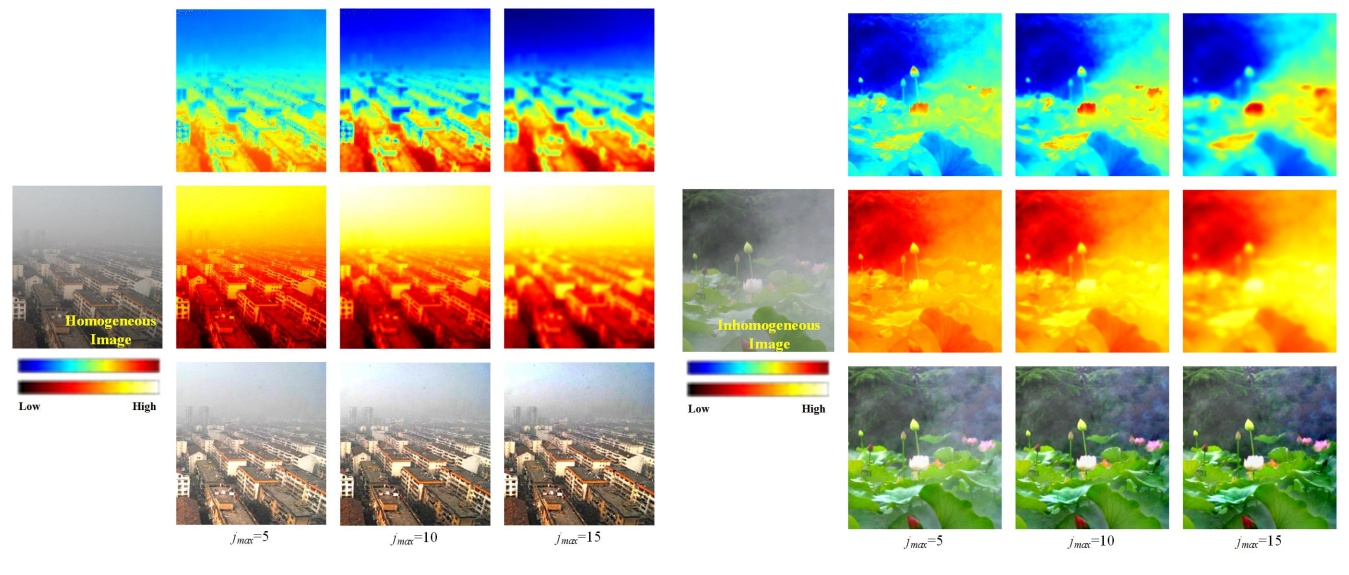

Fig. 3. Two dehazing examples of different types of hazy images using our BDPK by setting the $j_{\max } \in\{5,10,15\}$ with $\eta=0$. Top: The scattering distribution maps (Best viewed in color). Middle: The scene depth maps (Best viewed in color). Bottom: The restored haze-free images.

TABLE I

THE EMPIRICAL PARAMETERS OF DIFFERENT ATMOSPHERIC CONDITIONS USED IN BDPK

\begin{tabular}{|c|c|c|c|c|c|c|c|c|}
\hline Atmospheric condition & $\theta_{1}$ & $\theta_{2}$ & $\theta_{3}$ & $\theta_{4}$ & $\theta_{5}$ & $\theta_{6}$ & $\theta_{7}$ & $\theta_{8}$ \\
\hline Homogeneous & 3 & 0.1 & 0.5 & $0.5+(j-1)$ & 0.5 & 0.5 & $0.5+(j-1)$ & 0.5 \\
\hline Inhomogeneous & 3 & 0.3 & 0.5 & $0.5+2 \cdot(j-1)$ & 0.5 & 0.5 & $0.5+2 \cdot(j-1)$ & 0.5 \\
\hline
\end{tabular}

(17) is first separated into three independent sub-problems, which are expressed as

$$
\begin{gathered}
d=\operatorname{argmin}\left\{\theta_{1} \cdot\left\|\boldsymbol{I}^{\prime}-\boldsymbol{\rho}^{\prime}+\beta \cdot d\right\|_{2}^{2}+\theta_{3} \cdot\|d-\tilde{d}\|_{2}^{2}\right. \\
\left.+\theta_{4} \cdot\|\nabla d\|_{2}^{2}+\theta_{5} \cdot\|\nabla d-\nabla \bar{I}\|_{2}^{2}\right\} \\
\beta=\operatorname{argmin}\left\{\theta_{1} \cdot\left\|\boldsymbol{I}^{\prime}-\boldsymbol{\rho}^{\prime}+\beta \cdot d\right\|_{2}^{2}+\theta_{6} \cdot\|\beta-\tilde{\beta}\|_{2}^{2}\right. \\
\left.+\theta_{7} \cdot\|\nabla \beta\|_{2}^{2}+\theta_{8} \cdot\|\nabla \beta-\nabla \bar{I}\|_{2}^{2}\right\} \\
\rho^{\prime}=\operatorname{argmin}\left\{\theta_{1} \cdot\left\|\boldsymbol{I}^{\prime}-\boldsymbol{\rho}^{\prime}+\beta \cdot d\right\|_{2}^{2}\right. \\
\left.+\theta_{2} \cdot W \cdot\left\|\left(F-\nabla \boldsymbol{\rho}^{\prime}\right)\right\|_{2}^{2}\right\}
\end{gathered}
$$

To accelerate the calculation, the gradient approximation method [50] is selected to resolve sub-problems $(18) \sim(20)$. Then, their optimal solutions after the $j$ th iteration can be computed as

$$
d_{(j)}=\frac{-\theta_{1} \cdot \beta_{(j-1)} \circ M_{1}+\sum \cdot\left(\theta_{4}+\theta_{5}\right) \cdot M_{3}+\theta_{3} \cdot \tilde{d}+M_{5}}{\theta_{1} \circ \beta_{(j-1)} \circ \beta_{(j-1)}+\sum^{2} \cdot\left(\theta_{4}+\theta_{5}\right)+\theta_{3}}
$$

$\beta_{(j)}=\frac{-\theta_{1} \cdot d_{(j-1)} \circ M_{1}+\sum \cdot\left(\theta_{7}+\theta_{8}\right) \cdot M_{2}+\theta_{6} \cdot \tilde{\beta}+M_{6}}{\theta_{1} \circ d_{(j-1)} \circ d_{(j-1)}+\sum^{2} \cdot\left(\theta_{7}+\theta_{8}\right)+\theta_{6}}$

$$
\boldsymbol{\rho}_{(j)}^{\prime}=\frac{\theta_{1} \cdot\left(\boldsymbol{I}^{\prime}+\beta_{(j-1)} \circ d_{(j-1)}\right)+\theta_{2} \cdot \sum \cdot\left(\boldsymbol{M}_{4}+F\right)}{\theta_{1}+\theta_{2} \cdot \sum^{2} \cdot W}
$$

where $\boldsymbol{M}_{1}=\boldsymbol{I}^{\prime}-\boldsymbol{\rho}_{(j-1)}^{\prime}, M_{2}=\beta_{(j-1)} \otimes \Lambda, M_{3}=$ $d_{(j-1)} \otimes \Lambda, M_{4}=\boldsymbol{\rho}_{(j-1)}^{\prime} \otimes \Lambda, M_{5}=\theta_{5} \cdot \sum \cdot\left(\sum \cdot \boldsymbol{I}-\right.$ $\bar{I} \otimes \Lambda), \boldsymbol{M}_{6}=\theta_{8} \cdot \sum \cdot\left(\sum \cdot \boldsymbol{I}-\bar{I} \otimes \Lambda\right), \circ$ and $\otimes$ represent element-wise multiplication and convolution operator, respectively. $\Lambda=[010 ; 101 ; 010]$ is the convolution kernel and $\sum=4$ is the sum of elemental value in $\Lambda$. It should be noted that, the mathematical meaning of PDFs (14) (16) constructed using the structure prior are very similar to those of the guided image filter [14], the guided joint bilateral filter [36] and the guided total variation model [50]. However, the corresponding solution formulas (21) (22) are not the simple filtering process due to the interaction of each imaging parameter during the iteration procedure. Once the stop criterion $\left\|\boldsymbol{\rho}_{j}^{\prime}-\boldsymbol{\rho}_{j-1}^{\prime}\right\|_{1} /\left\|\boldsymbol{\rho}_{j}^{\prime}\right\|_{1} \leq \eta$ or the maximal number of iterations $j_{\max }$ is satisfied, the iteration is terminated and the scene albedo can be restored as

$$
\boldsymbol{\rho}(x, y)=\frac{\boldsymbol{S}(i)-e^{\boldsymbol{\rho}^{\prime}(x, y)}}{\boldsymbol{S}(i)}
$$

For clarity, the entire procedure of BDPK is summarized in Algorithm 1 (Please refer to Algorithm 1 for other details that we cannot cover in the text). To achieve a better balance between accuracy and efficiency, we empirically provide the parameters for different atmospheric conditions as listed in Table I. Fig. 3 shows two dehazing examples of homogeneous and inhomogeneous atmospheres using our BDPK by setting $j_{\max } \in\{5,10,15\}$ with $\eta=0$. As can be seen, increasing the number of iterations, more thoroughly removes the haze, and the scattering distribution map as well as scene depth are more in accordance with our intuitions. Interestingly, we do not state that the scattering value is correlated with altitude, even though the left scattering map estimated by BDPK in Fig. 3 obviously is affected by gravity [57]. This precise result illustrates that our BDPK has the ability to merge the advantages of all introduced priors by reasonably selecting a set of regular parameters. 


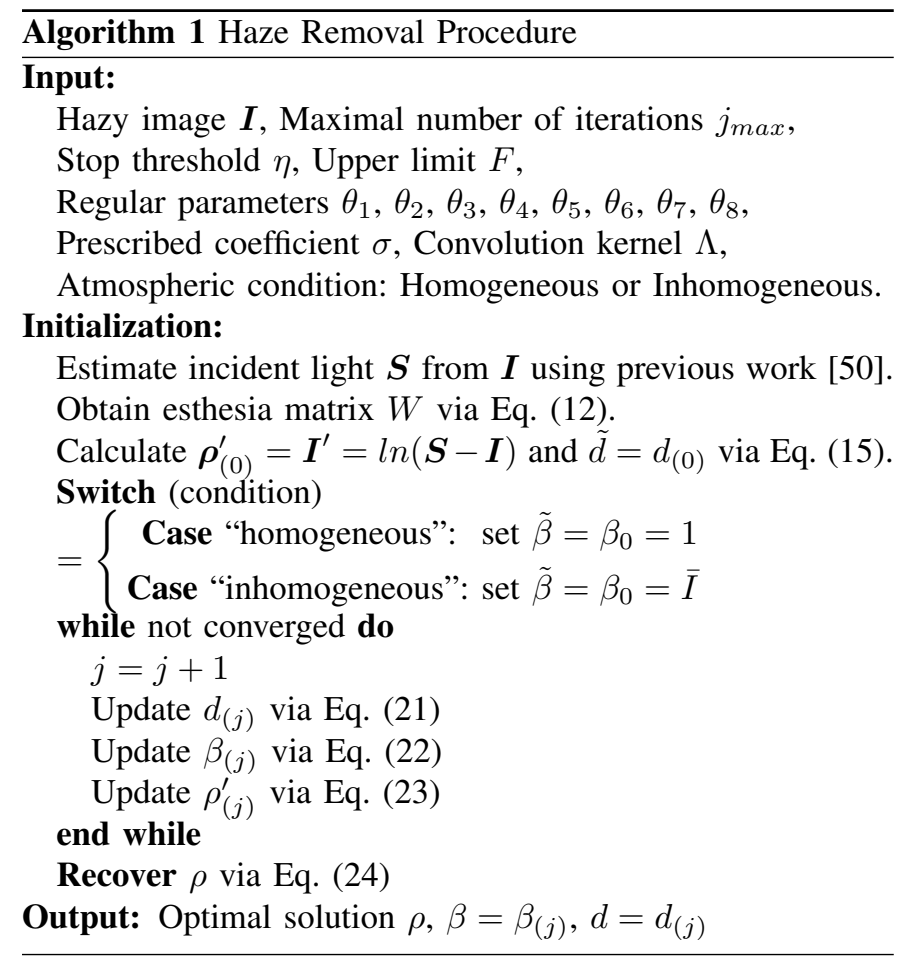

\section{EXPERIMENTAL COMPARISON AND ANALYSIS}

In this section, the relevance analyses of all priors introduced in BDPK are first illustrated experimentally. Next, we test BDPK on various hazy images and compare with classical image enhancement methods [6]-[8], [12]-[14] as well as the well-known techniques, including BD [49], BFCD [54], SIBF [55], OCE [41], MCP [22], MPE [19], FVR [58], DCP [25], SIIM [50], BCCR [37], CAP [42] and DN [44]. Among these techniques, the Bayesian-based $\mathrm{BD}, \mathrm{BFCD}$, and SIBF are selected for verifying the rationality of the optimization function designed in BDPK. The remaining methods qualitative and quantitative compared. The parameters involved in BDPK are initialized as follows: $j_{\max }=25, \eta=10^{-3}$, and the remainder are set as described in Section III (Please note that the initialization of $\tilde{\beta}$ requires user intervention). More test results and MATLAB code of BDPK are available on line: https://www.researchgate.net/profile/Can_Ding3/.

\section{A. Relevance Test of Introduced Priors}

In BDPK, three priori constraints are introduced for approximating the optimization function of haze removal. To check that all PDFs contained in BDPK can effectively be restrained to recover a realistic haze-free scene, we carry out the relevance test for BDPK by changing the regular parameters or redefining the PDF as a constant component. Fig. 4 shows the recovery results of BDPK with the different initialization states displayed in Table II. Through comparison, we find the model constraint prior is particularly important for BDPK due to its influence on the constrained solution space. The contrast prior [22] is used to highlight the visual clarity of the vague image. The structure prior [41], [50] imposes the scene depth and the scattering distribution has the efficacy to compensate for the interference of color distortion and remove the haze more thoroughly. It should be noted that although the structure prior used in BDPK is able to rectify the color distortion problem to a large extent (see Fig. $4 \mathrm{G}$ to $4 \mathrm{~J}$ ), the final result dehazed by BDPK shown in Fig. 4J still exhibits slight color shift. This is due to the fact that the incident light of each scene calculated via [50] would encounter estimation failure when the scene contains a bright target with large size. Meantime, the convolution operation employed in formulas (21-23) might introduce some halo artifacts into depth discontinuities of recovery results, but this interference is visually negligible and does not deteriorate the recovery quality of BDPK. In addition, two non-reference metrics are further employed to quantitatively assess the relevance results tested on real-world images, including the Fog Aware Density Evaluator (FADE) [52] and mean ratio of the gradients at the visible edges $\mathrm{R}$ [59]. In general, a smaller FADE leads to more complete haze removal. A higher $\mathrm{R}$ results in richer contrast information. The evaluated values for the dehazed results in Fig. 4 are listed in Table III. Analyzing these data, the outputs compromised via all priors achieve the best scores for the above-tested metrics.

\section{B. Example Results Using BDPK}

To validate the robustness of the proposed BDPK in this work, several types of hazy images are selected from previous research [24], [41], [50] and we process them with our BDPK. The corresponding recovery results shown in Fig. 5 greatly reveal the scenic structures in vague regions and improve the global contrast of hazy inputs, regardless of homogeneous and inhomogeneous weather conditions. Furthermore, BDPK is also equipped with the illumination compensation capability (ICC) (see the last group example). We believe the above superior performance mainly benefits from the universality of RASM and the accuracy of the optimization function designed in Section III.

\section{Qualitative Comparison with Image Enhancement Methods}

Because the traditional methods only blindly increase the textural contrast of degraded images, three hazy images that lack color information are picked for the subjective evaluation between the classic enhancement methods and our BDPK, as shown in Fig. 6 (a). Figs. 6(b-h) are the results restored by the histogram equalization [6], the adaptive histogram equalization [7], the Retinex method [9], the linear transformation [12], the homomorphic filtering [13], the Detail enhancement [14] and our BDPK, respectively. We observe that all the methods are able to locally or globally eliminate the haze from the hazy image to some extent. For the histogram equalization, linear transformation and homomorphic filtering, they have poorer restoration quality than other methods. It mainly reflects in the relatively dark (or bright) areas after processing might be saturated and thus loss the corresponding scenic details (see Figs. 6(b,e,f)). The results dehazed by Retinex method, as shown in Fig. 6(d), have clear scenic content in the misty parts. But, its dense hazy scenes still contain much haze or other unfavorable effects. In Figs. 6(c,g), the edge details become distinct remarkably, yet the dim color affects the visual quality 
TABLE II

THE REGULAR PARAMETERS USED IN BDPK FOR THE RECOVERY RESULTS SHOWN IN FIG. 4.

\begin{tabular}{|c|c|c|c|c|c|c|c|c|}
\hline Recovery Results & $\theta_{1}$ & $\theta_{2}$ & $\theta_{3}$ & $\theta_{4}$ & $\theta_{5}$ & $\theta_{6}$ & $\theta_{7}$ & $\theta_{8}$ \\
\hline Fig. 4A & 0 & 0.1 & 0.5 & $0.5+(j-1)$ & 0.5 & 0.5 & $0.5+(j-1)$ & 0.5 \\
\hline Fig. 4B & 3 & 0 & 0.5 & $0.5+(j-1)$ & 0.5 & 0.5 & $0.5+(j-1)$ & 0.5 \\
\hline \multirow{2}{*}{ Fig. 4C } & \multirow{2}{*}{3} & \multirow{2}{*}{0.1} & 0 & 0 & 0 & \multirow{2}{*}{0.5} & \multirow{2}{*}{$0.5+(j-1)$} & \multirow{2}{*}{0.5} \\
\hline & & & \multicolumn{3}{|c|}{ set $d=\tilde{d}$ directly } & & & \\
\hline \multirow{2}{*}{ Fig. 4D } & \multirow{2}{*}{3} & \multirow{2}{*}{0.1} & \multirow{2}{*}{0.5} & \multirow{2}{*}{$0.5+(j-1)$} & \multirow{2}{*}{0.5} & 0 & 0 & 0 \\
\hline & & & & & & \multicolumn{3}{|c|}{ set $\beta=\tilde{\beta}$ directly } \\
\hline Fig. 4E & 3 & 0.1 & 0.5 & $0.5+(j-1)$ & 0.5 & 0.5 & $0.5+(j-1)$ & 0.5 \\
\hline Fig. $4 \mathrm{~F}$ & 0 & 0.3 & 0.5 & $0.5+2 \cdot(j-1)$ & 0.5 & 0.5 & $0.5+2 \cdot(j-1)$ & 0.5 \\
\hline Fig. 4G & 3 & 0 & 0.5 & $0.5+2 \cdot(j-1)$ & 0.5 & 0.5 & $0.5+2 \cdot(j-1)$ & 0.5 \\
\hline \multirow{2}{*}{ Fig. $4 \mathrm{H}$} & \multirow{2}{*}{3} & \multirow{2}{*}{0.3} & 0 & 0 & 0 & \multirow{2}{*}{0.5} & \multirow{2}{*}{$0.5+2 \cdot(j-1)$} & \multirow{2}{*}{0.5} \\
\hline & & & & set $d=\tilde{d}$ directly & & & & \\
\hline \multirow{2}{*}{ Fig. 4I } & \multirow{2}{*}{3} & \multirow{2}{*}{0.3} & \multirow{2}{*}{0.5} & \multirow{2}{*}{$0.5+2 \cdot(j-1)$} & \multirow{2}{*}{0.5} & 0 & 0 & 0 \\
\hline & & & & & & \multicolumn{3}{|c|}{ set $\beta=\tilde{\beta}$ directly } \\
\hline Fig. $4 \mathrm{~J}$ & 3 & 0.3 & 0.5 & $0.5+2 \cdot(j-1)$ & 0.5 & 0.5 & $0.5+2 \cdot(j-1)$ & 0.5 \\
\hline
\end{tabular}

TABLE III

QUANTITATIVE PERFORMANCE COMPARISON OF THE RECOVERY RESULTS SHOWN IN FIG. 4 USING FADE AND R.

\begin{tabular}{|c|c|c|c|c|c|c|c|c|c|c|}
\hline Metric & Fig. 4A & Fig. 4B & Fig. 4C & Fig. 4D & Fig. 4E & Fig. 4F & Fig. 4G & Fig. 4H & Fig. 4I & Fig. 4J \\
\hline FADE & - & 0.5339 & 0.5178 & 0.4112 & $\mathbf{0 . 3 3 2 0}$ & - & 0.5963 & 0.3506 & 0.4202 & $\mathbf{0 . 2 6 8 5}$ \\
\hline R & - & 2.2285 & 2.0247 & 2.2196 & $\mathbf{2 . 7 6 9 5}$ & - & 1.6148 & 1.7261 & 1.4499 & $\mathbf{2 . 7 3 4 7}$ \\
\hline
\end{tabular}
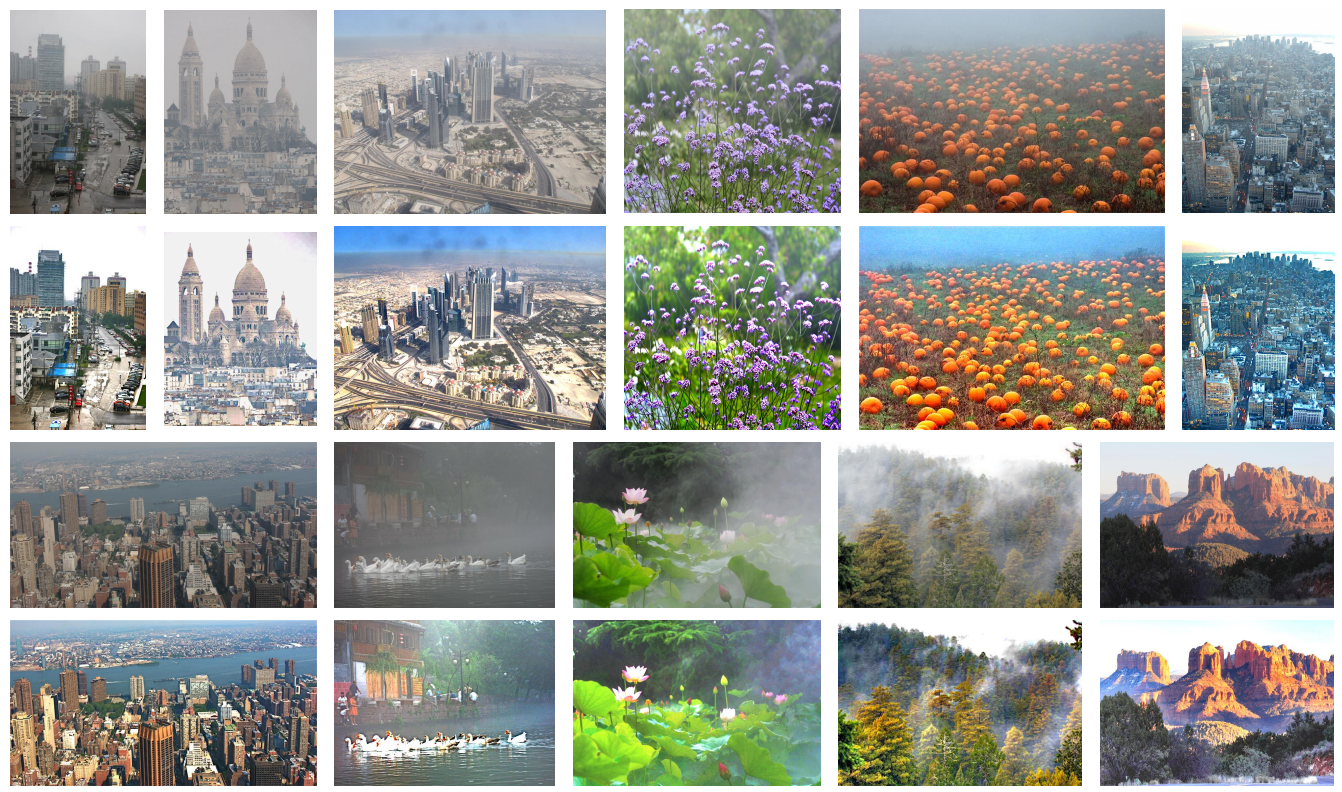

Fig. 5. Image dehazing results using BDPK. For each group: the above is the hazy image and the bottom is the restored result by BDPK.

of the dehazed images. In contrast, BDPK makes a good compensation for the brightness of low-illumination regions and perfectly uncovers the underlying target contour. Although the results dehazed by BDPK contain slight color distortion (the same reason as mentioned in Section IV-A), the restoration quality is better than other comparable enhancement methods.

\section{Qualitative Comparison with Bayesian-based Techniques}

To our best knowledge, there are three Bayesian-based methods for single image haze removal. They are BD [49], 


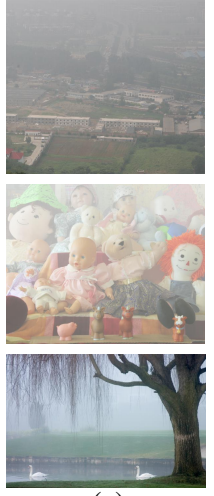

(a)

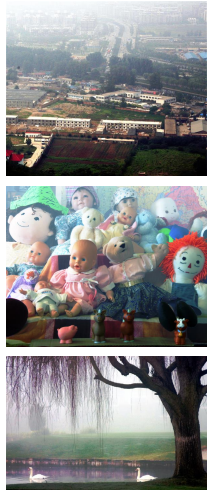

(b)

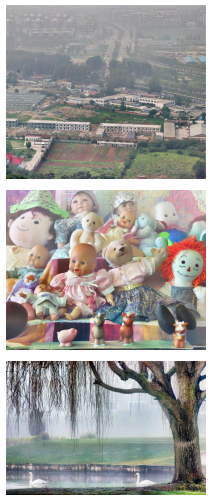

(c)

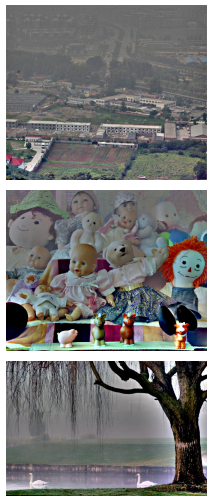

(d)

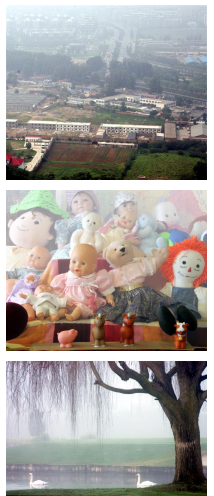

(e)

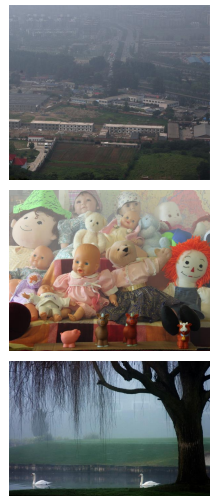

(f)

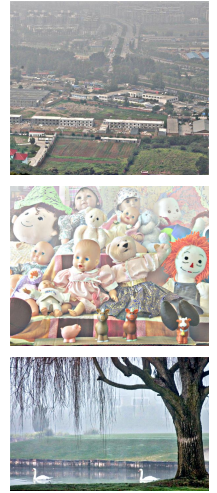

(g)

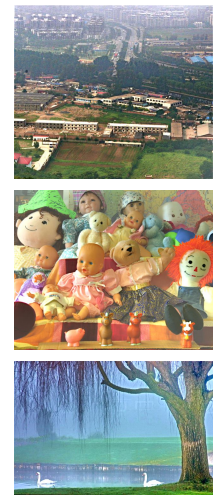

(h)

Fig. 6. Quantitative comparison between the traditional enhancement methods and our BDPK. (a) Hazy images. (b) Histogram equalization. (c) Adaptive histogram equalization. (d) Retinex method. (e) Linear transformation. (f) Homomorphic filtering. (g) Detail enhancement. (h) Our BDPK.

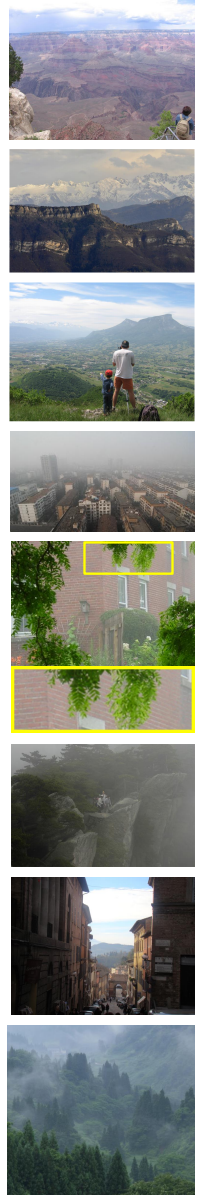

(a)
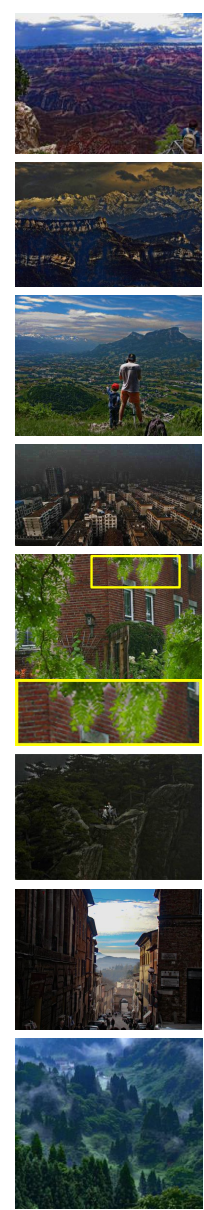

(b)

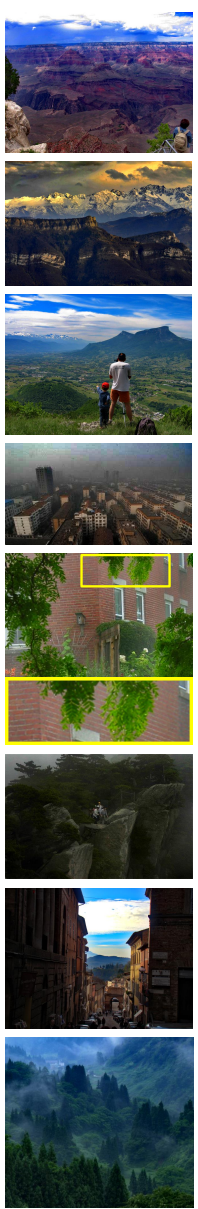

(c)

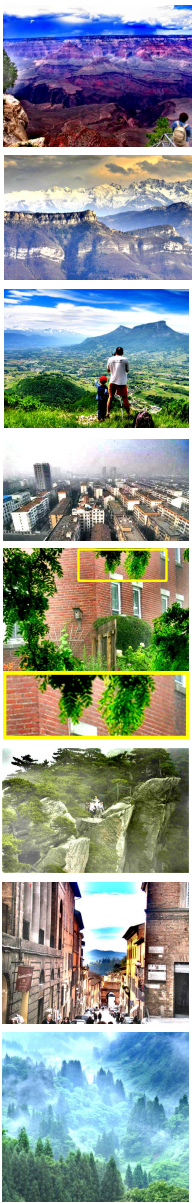

(d)

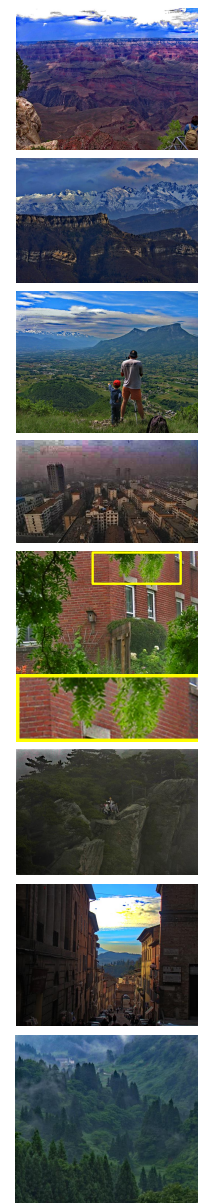

(e)

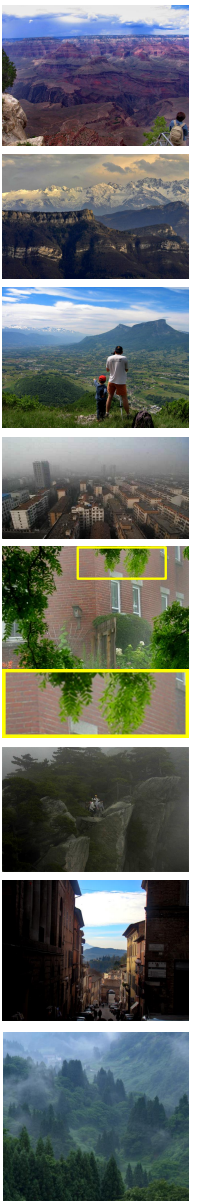

(f)

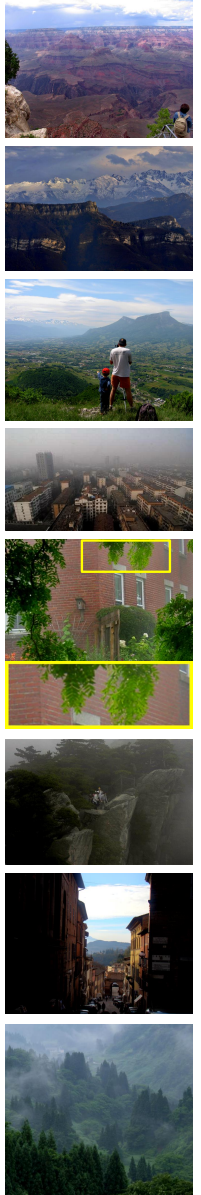

(g)
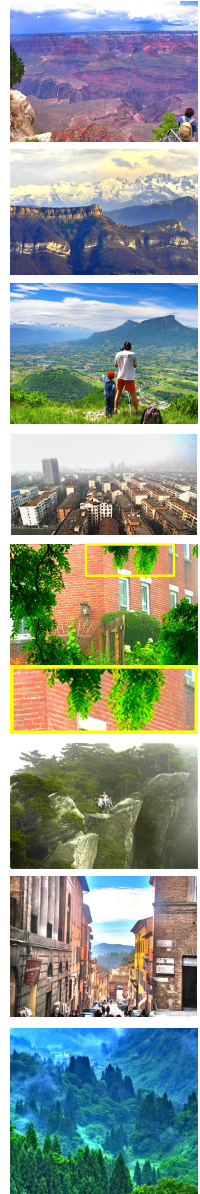

(h)

Fig. 9. Experimental results of different methods on eight real-world hazy images. (a) Hazy Image. (b) FVR's results. (c) DCP's results. (d) SIIM's results. (e) BCCR's results. (f) CAP's results. (g) DN's results. (h) BDPK's results.

BFCD [54] and SIBF [55]. The posterior probabilities used in BD were built using the gradient distribution prior and spatial smooth prior to restore the scene albedo with the canonical expectation maximization algorithm. Based on BD, BFCD further considered the noise factor into the degradation model and employed the BM3D [60] as well as the probability density function of transmission to simultaneously remove the haze and noise. In SIBF [55], the minimization function for restoring the degraded images was constructed by imposing the sparse prior on both the recovery result and the transmission map. To prove that the BDPK has better properties than the existing Bayesian-based works, the comparison between these similar methods [49], [54], [55] and BDPK is given in Fig. 7. Compared with the hazy image and the dehazed results (which are downloaded in [49], [54], [55]), the visual quality of each restored image shows different degrees of improvement, and 

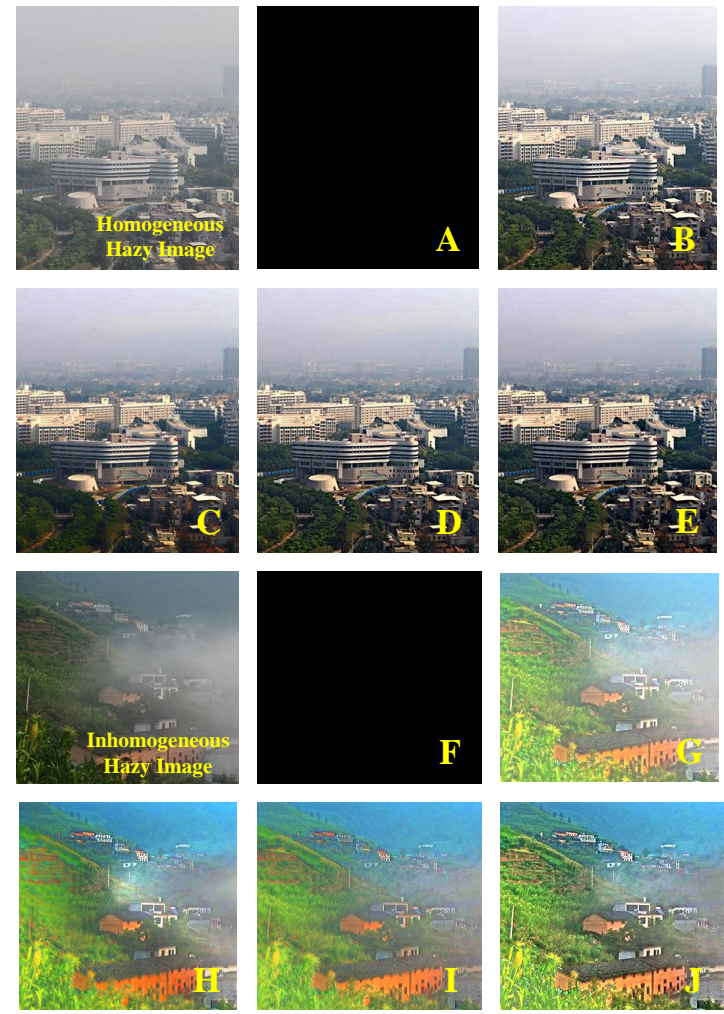

Fig. 4. The relevance test with different initialization states listed in Table II.

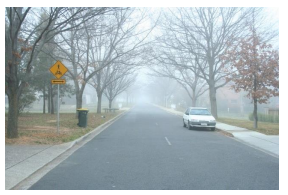

Hazy Image

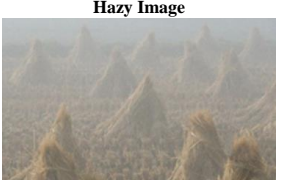

Hazy Image

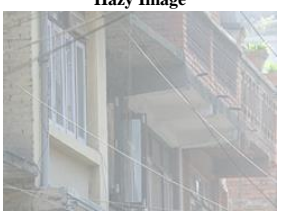

Hazy Image

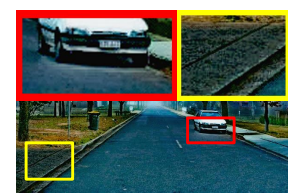

Result of BD

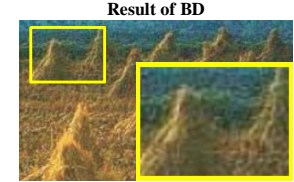

Result of BFCD

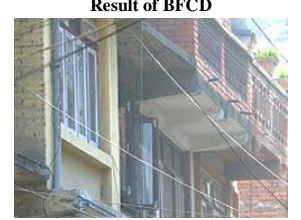

Result of SIBF

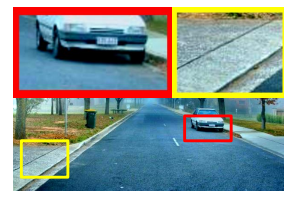

Result of our BDPK

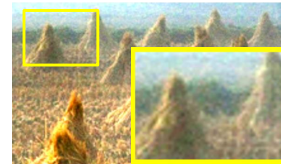

Result of our BDPK

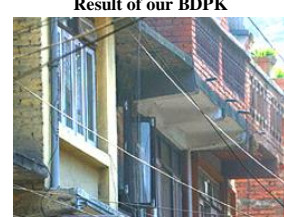

Result of our BDPK
Fig. 7. Comparison with the Bayesian-based works BD [49], BFCD [54] and SIBF [55].

all the methods can somewhat relieve the influence of haze. As shown in the first row in Fig. 7, BD can realize deep dehazing, but the over-enhancement often occurs in the flat areas that have slight textural details. In particular, the rocky areas in the recovered result are quite dark (see the yellow box). Likewise, due to the employed factorial Markov random fields [61], the halo artifact occurs in the red box. Similarly, BFCD suffers from over-saturation and produces much noise in the sky part (see the yellow box of the second row in Fig. 7). While SIBF rules out the interference of the above
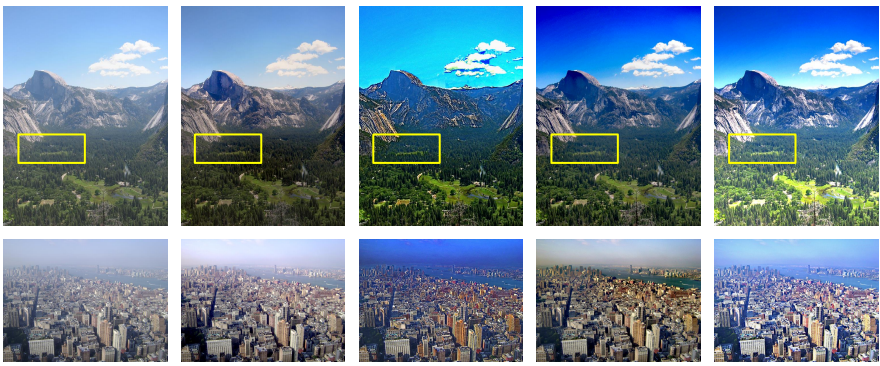

Fig. 8. Comparison with well-known image restoration techniques. From left to right: Hazy images, OCE's results, MCP's results, MPE's results, BDPK's results.

negative problems, the dehazing degree is visually weak (see the third row in Fig. 7). Conversely, BDPK's results have gentle sharpness and contrast, as well as the recovered color always looks very natural.

\section{E. Qualitative Comparison with Well-Known Techniques on Real-world Images}

1) First Group: In Fig. 8, OCE [41], MCP [22] and MPE [19] are processed with the two benchmark images. The reason that these methods were selected for comparison is that the recovery results are easily downloaded from Kim's websites: http://mcl.korea.ac.kr/projects/dehazing/. As we observed, OCE's results clearly show the object itself in the misty scene. However, since the control factor used in the cost function [41] is empirically set as a constant, the strength of the removed haze in the dense haze region is unstable compared to MCP, MPE and BDPK. For MCP, it can completely reveal the target contour for the given examples, yet the overenhancement was unfortunately introduced. Taking advantage of the given depth information, MPE successfully removes all the haze cover in the degenerated images. However, due to the constant atmospheric light defined in ASM, the visual clarity of recovered dark areas lacks competitiveness compared to BDPK (see the yellow box in the first row of Fig. 8). Through comparison, our resumed images are more distinct and clear, have a stronger sense of stereovision, and can address all of the above unfavorable effects.

2) Second Group: As is known, almost all the dehazing techniques are able to restore ideal results on general outdoor hazy images, it is very difficult to illustrate the superiority of the BDPK for restoration quality [42], [44]. Therefore, we focus on eight challenging real-world hazy images as the test samples of the second group, including the images with large gray scenes, misty haze, dense haze, uneven illumination and inhomogeneous atmosphere. Fig. 9 shows the qualitative comparison with six representative algorithms and BDPK on these challenging images. Fig. 9(a) depicts different types of hazy images to be dehazed, Figs. 9(b-h) show the restored results of FVR [58], DCP [25]+Guided Filter [14], SIIM [50], BCCR [37], CAP [42], DN [44] and BDPK, respectively. The recovery results corresponding to these representative methods shown in Fig. 9 are produced with the available MATLAB codes downloaded from the authors' websites in the MATLAB2010 environment on the same PC with Intel(R) 
Core(Tm) i5-4210U CPU @ 1.70GHz 8.00 GB RAM. This ensures the fair comparison.

As shown in Fig. 9(b), FVR recovers most of the scenic details and can be implemented in real time due to the linear complexity. However, FVR's results seriously suffer from over-enhancement since the inherent problem of overestimating the transmission is inevitable (see the second image). Furthermore, owed to the median filter with poor edgepreserving properties, the halo artifacts appear near the depth discontinuities (see the zoom-in patch of the fifth image). Although DCP has mostly promising results (see Fig. 9(c)), the color distortion still emerged in the sky regions (see the fourth image). This is since the accuracy of the estimated transmission mainly relies on the validity of DCP, which may be invalid when the scene's brightness is similar to the atmospheric light. For SIIM, this method could achieve impressive visual effects for most examples. However, its results might have adverse visual effects in dark regions with vague classification features (see the zoom-in patch of the fifth image in the Fig. 9 (d)). BCCR's results can fully eliminate the haze and unveil the scenic surface for all hazy targets. Regrettably, the dehazing results accompanied by over-saturation are bound to make the visual effect appear unrealistic. As we observe in Fig. 9(e), the rebuilt color in the sky region is significantly distorted, especially in the fourth and seventh images. In Fig. 9(f), although CAP avoids the halo artifacts and the overenhancement, the haze residual is obvious in the sixth image because simply regarding the scattering coefficient as a fixed constant may not suitable for all situations. As displayed the first four images in Fig. 9(g), DN is capable of generating the restored results with vivid color and necessary details. Nevertheless, it does not work well for dense hazy images, as the corresponding results often appear shrouded by a small amount of haze (see the sixth image). The reason might be explained as follows. The DehazeNet trained in [44] is designed only for transmission estimation and it lacks the contrast enhancement ability. On the other hand, the image pairs that are selected to train DehazeNet are artificially synthesized images rather than real-world images, which makes DehazeNet has a limited capability on dealing with real-world hazy images.

Apart from the above-mentioned problems, these dehazing techniques still share two common limitations: 1) These methods (except for SIIM) lack the ICC and thus cannot appropriately handle hazy images under uneven illumination (see the seventh row in Fig. 9) and 2) These methods may fail in the cases that the atmosphere is inhomogeneous (see the eighth row in Fig. 9). On the contrary, we notice that BDPK is completely unaffected by these limitations and can produce the realistic haze-free images while avoiding the halo artifacts, over-enhancement, over-saturation and the color distortion (see Fig. 9(h)).

\section{F. Comparison with Well-Known Techniques on Synthetic Im- ages}

1) Qualitative Comparison: Assessing the recovery techniques for a single hazy image is a difficult task because the haze-free reference images could not be provided in the real-world. To verify the utility on complete images, the six representative techniques and BDPK are compared on the synthesized hazy images collected in the D-HAZY dataset [62], in which the corresponding ground truth reference images are known. In Fig. 10, we show the results processed with different methods on these synthesized images. Fig. 10(a) depicts the hazy images with names 'Piano', 'Couch', 'Jadeplant', 'Room', 'Shelves', 'Sticks', 'Bicycle' and 'Vintage'. Fig. 10 (b) provides the ground truth images for fair comparison. Figs. 10(c-i) exhibit the results of FVR [58], DCP [25]]+Guided Filter [14]], SIIM [50], BCCR [37], CAP [42], DN [44] and BDPK, respectively.

As seen in Fig. 10(c), FVR's results usually look much darker than the ground truth images, and the halo artifacts can be found in the depth discontinuities (see the jadeplant image). For DCP, as shown in Fig. 10(d), the recovered images achieve a good compromise between haze removal degree and target clarity. However, the ground of the bicycle image shows its inaccuracies. In Figs. 10(e,f), the results restored by BCCR and SIIM are visually compelling, yet the restored colors might be more over-saturated than they should be, especially in the sticks image. By observing Figs. 10(g,h), we notice that CAP and DN are very positive for the given misty examples, while their dehazed results for the other hazy images are still surrounded by a small amount of mist (see the playroom image and piano image). Different from these techniques, BDPK's results do not possess the unpleasing effects and they maintain the original tones of the scene targets. This further illustrates the outstanding performance of BDPK compared to these techniques in terms of the human visual perspective.

2) Quantitative Comparison: To overcome the inevitable bias of qualitative comparisons and fairly assess these algorithms, we compute the non-reference metrics R, FADE as previously mentioned for the recovery results and choose three commonly used reference evaluation indicators as test criterion, namely structural similarity (SSIM) [63], mean squared error (MSE) and peak signal-to-noise ratio (PSNR). For these three metrics, a greater SSIM indicates better structural similarity between the dehazed result and ground truth image. A lower MSE and higher PSNR represent that the recovery results are more acceptable. Due to the unavailable haze-free scenes of the real-world hazy images, we only implement the quantitative comparison on the restoration

The quantitative assessments of the recovered results in Fig. 10 using SSIM, R, FADE, MSE and PSNR are summarized in Table IV. For the ease of measuring the effectiveness of different approaches, we have also calculated the mean, median and root mean square values of these scores over the set of images. Note that the best scores are highlighted with bold character in the table.

By analyzing the data, we notice that BDPK outperforms all the other methods for all the examples when assessed by SSIM, which indicates that BDPK produces the best image structures. For the metrics R and FADE, BDPK's scores are the best for the first five images, but they are not as good as FVR, SIIM, DCP and BCCR for the last three samples. However, this does not necessarily mean that other dehazing methods are better than BDPK. The reason they have a better score when 


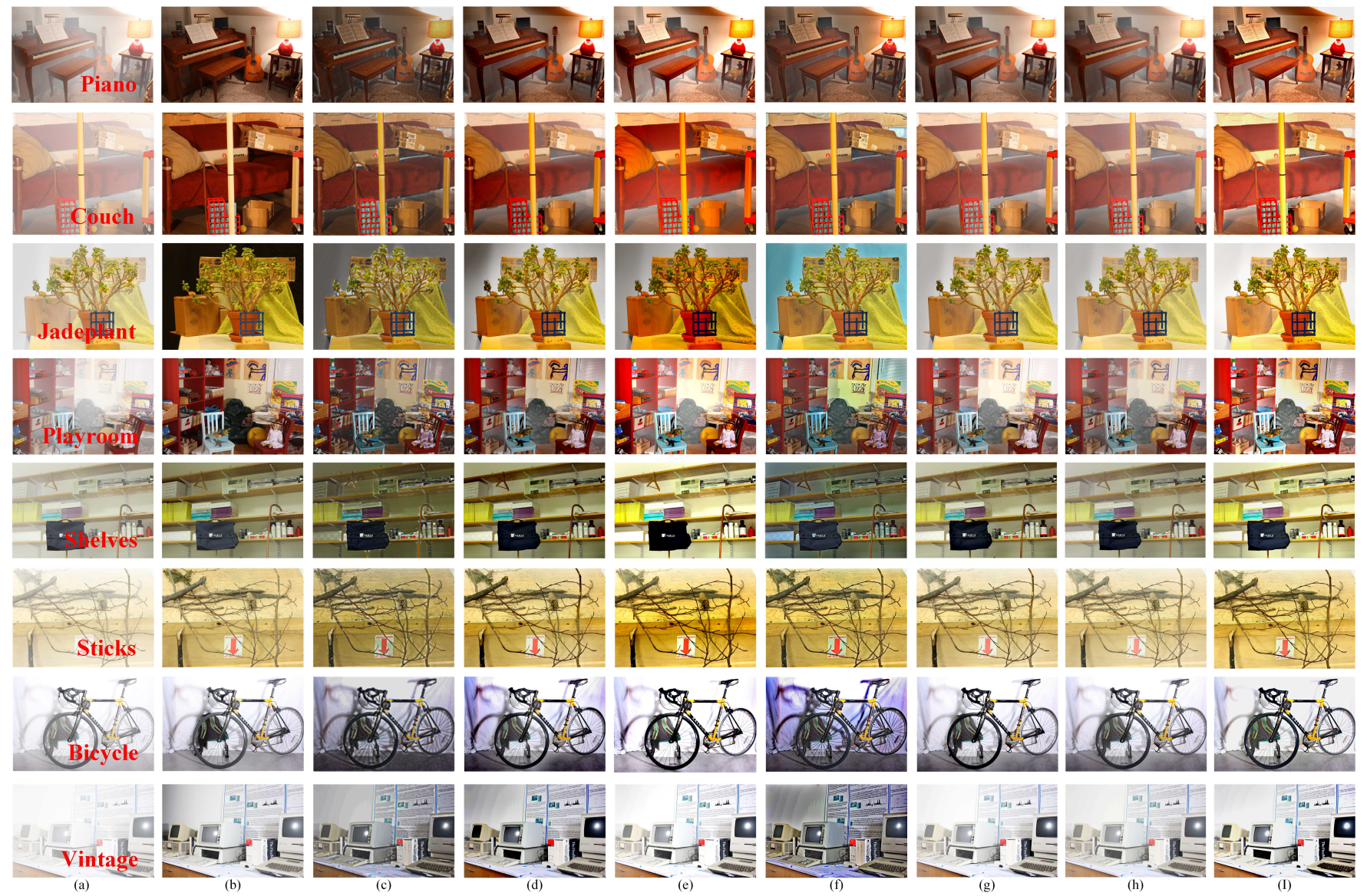

Fig. 10. Experimental results of different methods on eight synthesized images. (a) Hazy Image. (b) Ground Truth images. (c) FVR's results. (d) DCP's results. (e) SIIM's results. (f) BCCR's results. (g) CAP's results. (h) DN's results. (i) BDPK's results.

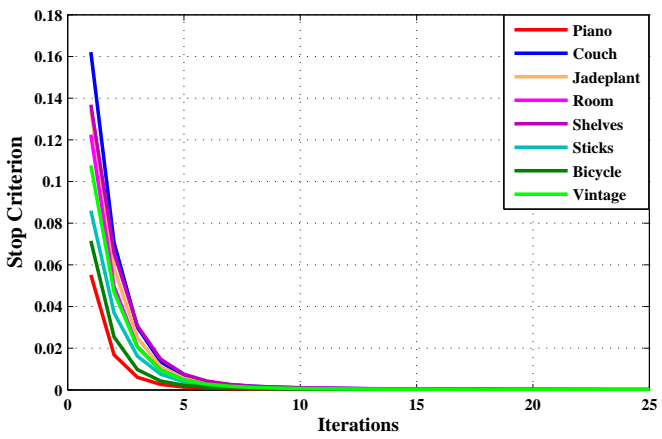

(a)

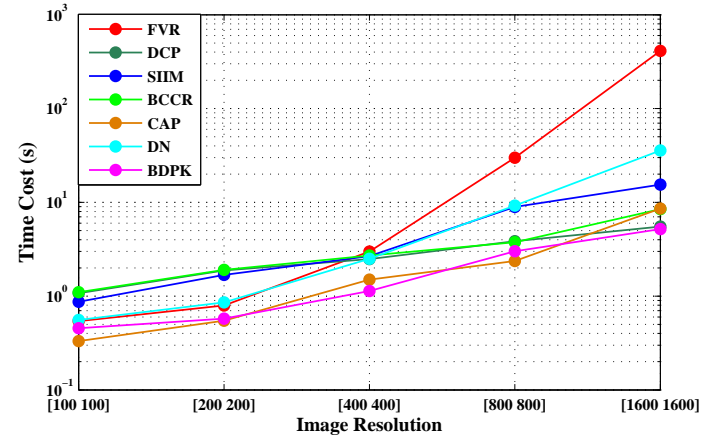

(b)

Fig. 11. (a): The convergence speed of the proposed BDPK in this work. The images used in this experiment are shown in Fig. 10. (b): Time comparison of different techniques with varying image sizes.

assessed by $\mathrm{R}$ and FADE is because the recovered results of these methods usually appear to be over-saturated or too dark. For example, BCCR's result (see Fig. 10(f)) on the bicycle image obtains the highest $R$ and FADE values, whereas almost all the negative effects can be visually observed. BDPK's MSE and PSNR scores are relatively lower than those of DCP and BCCR for dense hazy images (the first four examples) and are inferior to those of CAP and DN for misty images (the last four examples). Although DBPK does not have the best score for every example, the statistics results demonstrate that DBPK have the best performance on average.

\section{G. Complexity}

The computational complexity is another critical assessment factor for the dehazing technique. By analyzing Algorithm 1, we infer that the main computational overhead consumed in BDPK is the iteration module. Each iteration procedure mainly contains three convolution operations and other low-complex 
TABLE IV

QUANTITATIVE COMPARISON OF THE RECOVERED IMAGES IN FIG. 10 USING SSIM, R, FADE, MSE, PSNR.

\begin{tabular}{|c|c|c|c|c|c|c|c|c|}
\hline Metric & Examples & FVR & DCP & SIIM & BCCR & CAP & $\mathrm{DN}$ & BDPK \\
\hline \multirow{8}{*}{ SSIM } & Piano & 0.694 & 0.7367 & 0.6075 & 0.7555 & 0.7075 & 0.7081 & 0.8007 \\
\hline & Couch & 0.7176 & 0.7241 & 0.7271 & 0.7633 & 0.6568 & 0.6262 & 0.8247 \\
\hline & Jadeplant & 0.5625 & 0.5821 & 0.4939 & 0.5974 & 0.5688 & 0.549 & 0.6687 \\
\hline & Room & 0.6995 & 0.7772 & 0.7152 & 0.7722 & 0.7297 & 0.7154 & 0.7995 \\
\hline & Shelves & 0.7872 & 0.7788 & 0.7275 & 0.8611 & 0.835 & 0.884 & 0.8931 \\
\hline & Sticks & 0.8899 & 0.8618 & 0.7909 & 0.8677 & 0.9335 & 0.9412 & 0.9471 \\
\hline & Bicycle & 0.7282 & 0.7595 & 0.7659 & 0.6203 & 0.8303 & 0.8613 & 0.8743 \\
\hline & Vintage & 0.819 & 0.74 & 0.8348 & 0.7113 & 0.9409 & 0.9414 & 0.9498 \\
\hline \multicolumn{2}{|c|}{ SSIM Average } & 0.737238 & 0.745025 & 0.70785 & 0.7436 & 0.775313 & 0.778325 & 0.844738 \\
\hline \multicolumn{2}{|c|}{ SSIM Median } & 0.7229 & 0.74975 & 0.7273 & 0.7594 & 0.78 & 0.78835 & 0.8495 \\
\hline \multicolumn{2}{|c|}{ SSIM Root Mean Square } & 0.700392 & 0.705817 & 0.674245 & 0.706455 & 0.740216 & 0.745514 & 0.800608 \\
\hline \multirow{8}{*}{$\mathrm{R}$} & Piano & 1.8263 & 1.8263 & 2.1719 & 1.8713 & 1.5218 & 1.2195 & 2.4619 \\
\hline & Couch & 2.456 & 1.9693 & 2.5389 & 2.3699 & 1.7395 & 1.5637 & 2.5801 \\
\hline & Jadeplant & 1.5156 & 1.6296 & 1.7597 & 1.6056 & 1.2815 & 1.1199 & 1.7915 \\
\hline & Room & 2.2472 & 2.1 & 2.1072 & 2.339 & 1.4895 & 1.2848 & 2.4766 \\
\hline & Shelves & 1.6437 & 1.745 & 1.9907 & 1.9711 & 1.495 & 1.3616 & 2.0847 \\
\hline & Sticks & 2.6907 & 2.3316 & 2.9984 & 2.857 & 1.9161 & 1.6009 & 2.4916 \\
\hline & Bicycle & 2.4772 & 2.4874 & 2.1892 & 3.2258 & 1.9435 & 1.7443 & 2.2375 \\
\hline & Vintage & 4.0082 & 4.7389 & 3.1836 & 5.2087 & 2.3577 & 2.161 & 3.4917 \\
\hline \multicolumn{2}{|c|}{ R Average } & 2.358113 & 2.353513 & 2.36745 & 2.68105 & 1.718075 & 1.506963 & 2.45195 \\
\hline & Median & 2.3516 & 2.03465 & 2.18055 & 2.35445 & 1.63065 & 1.46265 & 2.46925 \\
\hline \multicolumn{2}{|c|}{ R Root Mean Square } & 2.329951 & 2.389905 & 2.275014 & 2.723378 & 1.648063 & 1.451687 & 2.352618 \\
\hline \multirow{8}{*}{ FADE } & Piano & 0.3271 & 0.2568 & 0.3543 & 0.3166 & 0.4899 & 0.5889 & 0.2379 \\
\hline & Couch & 0.285 & 0.3394 & 0.268 & 0.3161 & 0.4934 & 0.6156 & 0.2516 \\
\hline & Jadeplant & 0.4404 & 0.2859 & 0.2094 & 0.3284 & 0.6024 & 0.7437 & 0.3704 \\
\hline & Room & 0.258 & 0.2128 & 0.2828 & 0.2413 & 0.4854 & 0.5824 & 0.2092 \\
\hline & Shelves & 0.3124 & 0.2562 & 0.3877 & 0.2762 & 0.3992 & 0.6185 & 0.2437 \\
\hline & Sticks & 0.1958 & 0.2369 & 0.1732 & 0.1995 & 0.5011 & 0.6203 & 0.2148 \\
\hline & Bicycle & 0.3323 & 0.327 & 0.4378 & 0.2652 & 0.4777 & 0.6333 & 0.4144 \\
\hline & Vintage & 0.6306 & 0.3305 & 0.863 & 0.2674 & 1.1127 & 1.2393 & 0.4767 \\
\hline \multicolumn{2}{|c|}{ FADE Average } & 0.3477 & 0.280688 & 0.372025 & 0.276338 & 0.570225 & 0.70525 & 0.314838 \\
\hline \multicolumn{2}{|c|}{ FADE Median } & 0.31975 & 0.27135 & 0.31855 & 0.2708 & 0.49165 & 0.6194 & 0.29765 \\
\hline \multirow{2}{*}{\multicolumn{2}{|c|}{\begin{tabular}{c|c}
\multicolumn{2}{|c|}{ FADE Root Mean Square } \\
Piano
\end{tabular}}} & 0.369585 & 0.284183 & 0.423958 & 0.279318 & 0.608143 & 0.735042 & 0.328652 \\
\hline & & 0.0327 & 0.0154 & 0.0828 & 0.0155 & 0.0365 & 0.0336 & 0.0203 \\
\hline \multirow{7}{*}{ MSE } & Couch & 0.0585 & 0.0298 & 0.0386 & 0.0238 & 0.0653 & 0.0793 & 0.0308 \\
\hline & Jadeplant & 0.1354 & 0.1216 & 0.1384 & 0.1162 & 0.1785 & 0.2035 & 0.1234 \\
\hline & Room & 0.0482 & 0.0183 & 0.0562 & 0.0178 & 0.0466 & 0.0488 & 0.0294 \\
\hline & Shelves & 0.0323 & 0.0213 & 0.03 & 0.0216 & 0.011 & 0.015 & 0.0206 \\
\hline & Sticks & 0.031 & 0.0203 & 0.0343 & 0.0165 & 0.0075 & 0.0077 & 0.0092 \\
\hline & Bicycle & 0.0751 & 0.0444 & 0.0228 & 0.0857 & 0.0167 & 0.0105 & 0.0213 \\
\hline & Vintage & 0.0457 & 0.0536 & 0.0287 & 0.054 & 0.0165 & 0.0122 & 0.0185 \\
\hline \multicolumn{2}{|c|}{ MSE Average } & 0.057363 & 0.040588 & 0.053975 & 0.043888 & 0.047325 & 0.051325 & $\mathbf{0 . 0 3 4 1 8 8}$ \\
\hline \multicolumn{2}{|c|}{ MSE Median } & 0.04695 & 0.02555 & 0.03645 & 0.0227 & 0.0266 & 0.0243 & 0.02095 \\
\hline \multicolumn{2}{|c|}{ MSE Root Mean Square } & 0.066031 & 0.052389 & 0.065268 & 0.056596 & 0.071027 & 0.080432 & 0.048422 \\
\hline \multirow{8}{*}{ PSNR } & Piano & 14.8582 & 18.1232 & 10.8219 & 18.0832 & 14.3762 & 14.7383 & 16.4392 \\
\hline & Couch & 12.4006 & 15.2578 & 14.1356 & 16.239 & 11.8494 & 11.0082 & 15.1177 \\
\hline & Jadeplant & 8.7484 & 9.1501 & 8.5902 & 9.3464 & 7.4848 & 6.9138 & 9.0889 \\
\hline & Room & 13.3944 & 17.3765 & 12.5034 & 17.4888 & 13.3157 & 13.1201 & 15.3198 \\
\hline & Shelves & 14.905 & 16.6521 & 15.2349 & 16.3373 & 19.5694 & 18.2491 & 17.2228 \\
\hline & Sticks & 15.09 & 16.9328 & 14.6498 & 17.8224 & 21.1166 & 21.0534 & 20.3731 \\
\hline & Bicycle & 11.2459 & 13.5309 & 16.428 & 10.6685 & 17.7772 & 19.7946 & 16.7214 \\
\hline & Vintage & 13.4043 & 12.7071 & 15.414 & 12.6784 & 17.8318 & 19.144 & 17.3395 \\
\hline \multirow{2}{*}{\multicolumn{2}{|c|}{$\begin{array}{l}\text { PSNR Average } \\
\text { PSNR Median }\end{array}$}} & 13.00585 & 14.96631 & 13.47223 & 14.833 & 15.41514 & 15.51519 & 15.9528 \\
\hline & & 13.39935 & 15.95495 & 14.3927 & 16.28815 & 16.0767 & 16.4937 & 16.5803 \\
\hline 1 & ot Mean Square & 13.16481 & 15.22944 & 13.69874 & 15.17821 & 15.98158 & 16.19086 & 16.23287 \\
\hline
\end{tabular}

operations (see Equations. (21-23)). Ignoring these simple calculations, for the given image with resolution res and the determined convolution kernel $\Lambda$, the theoretical complexity of BDPK is only $O\left(\right.$ res $\left.\cdot|\Lambda| \cdot j_{a}\right)(|\Lambda|$ is the number of elements in $\Lambda$ and $j_{a}$ is the actual iteration number). Despite this, it may be more intuitive to see how many iterations are needed to empirically converge. Fig. 11(a) plots eight iteration curves for the images shown in Fig. 10. From these plots, we notice that the changing trends of the eight plots are similar to each other, and the actual iteration number is approximately 10 iterations for all tests. Fig. 11(b) gives the comparison between FVR [58], DCP [25] + Guided Filter [35], SIIM [50], BCCR [37], CAP [42], DN [44] and our BDPK in terms of time costs (In the same PC described above, all the calculation times are recorded by running the authors' codes or the projects simulated by us on the hazy images with different resolutions). As we expected, the BDPK proposed in this work is much faster than FVR, DN, SIIM, DCP and BCCR, as well as is very close to the CAP with high efficiency. Even when the given hazy image is very large, BDPK still achieves high- 
speed processing.

\section{Discussion AND CONCLUSION}

In this paper, we have redefined a novel atmospheric scattering model (RASM) according to our previous work [50] and the observation of atmospheric particles distribution. Afterwards, a single image dehazing algorithm called BDPK was further proposed based on this RASM. Unlike previous works, the key contribution of our BDPK is to convert the haze removal into an optimization function by combining Bayesian theory and prior knowledge. This allows us to directly recover the scene albedo with an alternating minimizing technique (AMT). Experimental results demonstrate the superiority of the proposed BDPK in terms of the dehazing effectiveness and the robustness compared with the state-of-the-art techniques.

Although we have designed an effective and robust dehazing method, there are some future studies that should be conducted. Atmospheric conditions are required to be artificially determined in our BDPK. Therefore, seeking an adaptive approach to detect the atmospheric conditions in hazy images is of practical significance. Considering that the weight coefficients involved in AMT are empirically determined, we will develop a learning-based strategy to pursue a set of optimal coefficients that can achieve a better balance between dehazing quality and computational efficiency.

\section{ACKNOWLEDGMENT}

This study was supported in part by the National Natural Science Foundation of China [61571241] [61872423], Industry-university-research Prospective Joint Project of Jiangsu Province [BY2014014], Major Projects of Jiangsu Province University Natural Science Research [15KJA510002] and Research Innovation Program for College Graduates of Jiangsu Province [KYLX16_0665].

\section{REFERENCES}

[1] I. Haritaoglu, D. Harwood, and L. S. Davis, "W4: real-time surveillance of people and their activities," IEEE Transactions on Pattern Analysis and Machine Intelligence, vol. 22, no. 8, pp. 809-830, Aug 2000.

[2] S. Coar, G. Donatiello, V. Bogorny, C. Garate, L. O. Alvares, and F. Brmond, "Toward abnormal trajectory and event detection in video surveillance," IEEE Transactions on Circuits and Systems for Video Technology, vol. 27, no. 3, pp. 683-695, March 2017.

[3] Y. Dong, Z. Hu, K. Uchimura, and N. Murayama, "Driver inattention monitoring system for intelligent vehicles: A review," IEEE Transactions on Intelligent Transportation Systems, vol. 12, no. 2, pp. 596-614, June 2011.

[4] P. F. Felzenszwalb and D. P. Huttenlocher, "Pictorial structures for object recognition," International journal of computer vision, vol. 61, no. 1, pp. 55-79, 2005.

[5] S. Belongie, J. Malik, and J. Puzicha, "Shape matching and object recognition using shape contexts," IEEE Transactions on Pattern Analysis and Machine Intelligence, vol. 24, no. 4, pp. 509-522, Apr 2002.

[6] W. Frei, "Image enhancement by histogram hyperbolization," Computer Graphics and Image Processing, vol. 6, no. 3, pp. 286-294, 1977.

[7] S. M. Pizer, E. P. Amburn, J. D. Austin, R. Cromartie, A. Geselowitz, T. Greer, B. ter Haar Romeny, J. B. Zimmerman, and K. Zuiderveld, "Adaptive histogram equalization and its variations," Computer vision, graphics, and image processing, vol. 39, no. 3, pp. 355-368, 1987.

[8] D. J. Jobson, Z. Rahman, and G. A. Woodell, "A multiscale retinex for bridging the gap between color images and the human observation of scenes," IEEE Transactions on Image Processing, vol. 6, no. 7, pp. 965-976, Jul 1997.
[9] B. Funt, F. Ciurea, and J. McCann, "Retinex in matlab," Journal of electronic imaging, vol. 13, no. 1, pp. 48-57, 2004.

[10] Z.-u. Rahman, G. A. Woodell, and D. J. Jobson, "A comparison of the multiscale retinex with other image enhancement techniques," 1997.

[11] S. C. Huang, F. C. Cheng, and Y. S. Chiu, "Efficient contrast enhancement using adaptive gamma correction with weighting distribution," IEEE Transactions on Image Processing, vol. 22, no. 3, pp. 1032-1041, March 2013.

[12] J. Weickert, "A review of nonlinear diffusion filtering," Scale-space theory in computer vision, pp. 1-28, 1997.

[13] M.-J. Seow and V. K. Asari, "Ratio rule and homomorphic filter for enhancement of digital colour image," Neurocomputing, vol. 69, no. 7, pp. 954-958, 2006.

[14] K. He, J. Sun, and X. Tang, "Guided image filtering," IEEE Transactions on Pattern Analysis and Machine Intelligence, vol. 35, no. 6, pp. 13971409, June 2013

[15] L. Cox, "Optics of the atmosphere-scattering by molecules and particles," Journal of Modern Optics, vol. 24, no. 7, pp. 779-779, 1977.

[16] W. Wang, X. Yuan, X. Wu, and Y. Liu, "Fast image dehazing method based on linear transformation," IEEE Transactions on Multimedia, vol. 19 , no. 6, pp. 1142-1155, June 2017

[17] E. J. McCartney, "Optics of the atmosphere: scattering by molecules and particles," New York, John Wiley and Sons, Inc., 1976. 421 p., 1976.

[18] S. G. Narasimhan and S. K. Nayar, "Contrast restoration of weather degraded images," IEEE Transactions on Pattern Analysis and Machine Intelligence, vol. 25, no. 6, pp. 713-724, June 2003.

[19] J. Kopf, B. Neubert, B. Chen, M. Cohen, D. Cohen-Or, O. Deussen, M. Uyttendaele, and D. Lischinski, "Deep photo: Model-based photograph enhancement and viewing," in ACM transactions on graphics (TOG), vol. 27, no. 5. ACM, 2008, p. 116.

[20] Y. Y. Schechner, S. G. Narasimhan, and S. K. Nayar, "Instant dehazing of images using polarization," in Computer Vision and Pattern Recognition, 2001. CVPR 2001. Proceedings of the 2001 IEEE Computer Society Conference on, vol. 1. IEEE, 2001, pp. I-I.

[21] S. Shwartz, E. Namer, and Y. Y. Schechner, "Blind haze separation," in 2006 IEEE Computer Society Conference on Computer Vision and Pattern Recognition (CVPR'06), vol. 2, 2006, pp. 1984-1991.

[22] R. T. Tan, "Visibility in bad weather from a single image," in 2008 IEEE Conference on Computer Vision and Pattern Recognition, June 2008, pp. $1-8$.

[23] R. Fattal, "Single image dehazing," ACM transactions on graphics (TOG), vol. 27, no. 3, p. 72, 2008.

[24] - "Dehazing using color-lines," ACM transactions on graphics (TOG), vol. 34, no. 1, p. 13, 2014

[25] K. He, J. Sun, and X. Tang, "Single image haze removal using dark channel prior," IEEE Transactions on Pattern Analysis and Machine Intelligence, vol. 33, no. 12, pp. 2341-2353, Dec 2011.

[26] A. Levin, D. Lischinski, and Y. Weiss, "A closed-form solution to natural image matting," IEEE Transactions on Pattern Analysis and Machine Intelligence, vol. 30, no. 2, pp. 228-242, Feb 2008.

[27] B.-H. Chen and S.-C. Huang, "Edge collapse-based dehazing algorithm for visibility restoration in real scenes," Journal of Display Technology, vol. 12, no. 9, pp. 964-970, 2016.

[28] D. K. Jha, B. Gupta, and S. S. Lamba, "1 2-norm-based prior for hazeremoval from single image," IET Computer Vision, vol. 10, no. 5, pp. $331-341,2016$

[29] S. C. Huang, J. H. Ye, and B. H. Chen, "An advanced single-image visibility restoration algorithm for real-world hazy scenes," IEEE Transactions on Industrial Electronics, vol. 62, no. 5, pp. 2962-2972, May 2015.

[30] B.-H. Chen and S.-C. Huang, "An advanced visibility restoration algorithm for single hazy images," ACM Transactions on Multimedia Computing, Communications, and Applications (TOMM), vol. 11, no. 4, p. $53,2015$.

[31] L. Zeng and Y. Dai, "Single image dehazing based on combining dark channel prior and scene radiance constraint," Chinese Journal of Electronics, vol. 25, no. 6, pp. 1114-1120, 2016.

[32] S. C. Huang, B. H. Chen, and Y. J. Cheng, "An efficient visibility enhancement algorithm for road scenes captured by intelligent transportation systems," IEEE Transactions on Intelligent Transportation Systems, vol. 15, no. 5, pp. 2321-2332, Oct 2014.

[33] K. B. Gibson, D. T. Vo, and T. Q. Nguyen, "An investigation of dehazing effects on image and video coding," IEEE Transactions on Image Processing, vol. 21, no. 2, pp. 662-673, Feb 2012.

[34] S. C. Huang, B. H. Chen, and W. J. Wang, "Visibility restoration of single hazy images captured in real-world weather conditions," IEEE 
Transactions on Circuits and Systems for Video Technology, vol. 24, no. 10, pp. 1814-1824, Oct 2014.

[35] J. Yu, C. Xiao, and D. Li, "Physics-based fast single image fog removal," in IEEE 10th INTERNATIONAL CONFERENCE ON SIGNAL PROCESSING PROCEEDINGS, Oct 2010, pp. 1048-1052.

[36] C. Xiao and J. Gan, "Fast image dehazing using guided joint bilateral filter," The Visual Computer, vol. 28, no. 6-8, pp. 713-721, 2012.

[37] G. Meng, Y. Wang, J. Duan, S. Xiang, and C. Pan, "Efficient image dehazing with boundary constraint and contextual regularization," in 2013 IEEE International Conference on Computer Vision, Dec 2013, pp. 617-624.

[38] B.-H. Chen, S.-C. Huang, and J. H. Ye, "Hazy image restoration by bihistogram modification," ACM Transactions on Intelligent Systems and Technology (TIST), vol. 6, no. 4, p. 50, 2015.

[39] L. He, J. Zhao, N. Zheng, and D. Bi, "Haze removal using the differencestructure-preservation prior," IEEE Transactions on Image Processing, vol. 26, no. 3, pp. 1063-1075, March 2017.

[40] Y. H. Lai, Y. L. Chen, C. J. Chiou, and C. T. Hsu, "Single-image dehazing via optimal transmission map under scene priors," IEEE Transactions on Circuits and Systems for Video Technology, vol. 25, no. 1, pp. 1-14, Jan 2015.

[41] J.-H. Kim, W.-D. Jang, J.-Y. Sim, and C.-S. Kim, "Optimized contrast enhancement for real-time image and video dehazing," Journal of Visual Communication and Image Representation, vol. 24, no. 3, pp. 410-425, 2013.

[42] Q. Zhu, J. Mai, and L. Shao, "A fast single image haze removal algorithm using color attenuation prior," IEEE Transactions on Image Processing, vol. 24, no. 11, pp. 3522-3533, Nov 2015

[43] K. Tang, J. Yang, and J. Wang, "Investigating haze-relevant features in a learning framework for image dehazing," in 2014 IEEE Conference on Computer Vision and Pattern Recognition, June 2014, pp. 2995-3002.

[44] B. Cai, X. Xu, K. Jia, C. Qing, and D. Tao, "Dehazenet: An end-to-end system for single image haze removal," IEEE Transactions on Image Processing, vol. 25, no. 11, pp. 5187-5198, Nov 2016.

[45] W. Ren, S. Liu, H. Zhang, J. Pan, X. Cao, and M.-H. Yang, "Single image dehazing via multi-scale convolutional neural networks," in $E u$ ropean Conference on Computer Vision. Springer, 2016, pp. 154-169.

[46] A. Galdran, J. Vazquez-Corral, D. Pardo, and M. Bertalmío, "Enhanced variational image dehazing," SIAM Journal on Imaging Sciences, vol. 8, no. 3, pp. 1519-1546, 2015.

[47] V. W. De Dravo and J. Y. Hardeberg, "Stress for dehazing," in Colour and Visual Computing Symposium (CVCS), 2015. IEEE, 2015, pp. 1-6.

[48] A. Galdran, J. Vazquez-Corral, D. Pardo, and M. Bertalmo, "Fusionbased variational image dehazing," IEEE Signal Processing Letters, vol. 24, no. 2, pp. 151-155, Feb 2017.

[49] K. Nishino, L. Kratz, and S. Lombardi, "Bayesian defogging," International journal of computer vision, vol. 98, no. 3, pp. 263-278, 2012.

[50] M. Ju, D. Zhang, and X. Wang, "Single image dehazing via an improved atmospheric scattering model," The Visual Computer, vol. 33, no. 12, pp. 1613-1625, 2017.

[51] C. O. Ancuti and C. Ancuti, "Single image dehazing by multi-scale fusion," IEEE Transactions on Image Processing, vol. 22, no. 8, pp. 3271-3282, Aug 2013.

[52] L. K. Choi, J. You, and A. C. Bovik, "Referenceless prediction of perceptual fog density and perceptual image defogging," IEEE Transactions on Image Processing, vol. 24, no. 11, pp. 3888-3901, Nov 2015.

[53] L. Kratz and K. Nishino, "Factorizing scene albedo and depth from a single foggy image," in 2009 IEEE 12th International Conference on Computer Vision, Sept 2009, pp. 1701-1708.

[54] D. Nan, D.-y. Bi, C. Liu, S.-p. Ma, and L.-y. He, "A bayesian framework for single image dehazing considering noise," The Scientific World Journal, vol. 2014, 2014.

[55] W. Duochao, W. Yongguo, D. Xuemei, H. Xi-Yuan, and P. Si-Long, "Single image dehazing based on bayesian framework," Journal of Computer-Aided Design \& Computer Graphics, vol. 22, no. 10, pp. 1756-1761, 2010.

[56] J. Pan, D. Sun, H. Pfister, and M. H. Yang, "Blind image deblurring using dark channel prior," in 2016 IEEE Conference on Computer Vision and Pattern Recognition (CVPR), June 2016, pp. 1628-1636.

[57] Z. Shi, J. Long, W. Tang, and C. Zhang, "Single image dehazing in inhomogeneous atmosphere," Optik-international journal for light and electron optics, vol. 125, no. 15, pp. 3868-3875, 2014.

[58] J. P. Tarel and N. Hautire, "Fast visibility restoration from a single color or gray level image," in 2009 IEEE 12th International Conference on Computer Vision, Sept 2009, pp. 2201-2208.
[59] N. Hautière, J.-P. Tarel, D. Aubert, and E. Dumont, "Blind contrast enhancement assessment by gradient ratioing at visible edges," Image Analysis \& Stereology, vol. 27, no. 2, pp. 87-95, 2011.

[60] K. Dabov, A. Foi, V. Katkovnik, and K. Egiazarian, "Bm3d image denoising with shape-adaptive principal component analysis," in SPARS'09-Signal Processing with Adaptive Sparse Structured Representations, 2009.

[61] J. Kim and R. Zabih, "Factorial markov random fields," in European Conference on Computer Vision. Springer, 2002, pp. 321-334.

[62] C. Ancuti, C. O. Ancuti, and C. De Vleeschouwer, "D-hazy: A dataset to evaluate quantitatively dehazing algorithms," in Image Processing (ICIP), 2016 IEEE International Conference on. IEEE, 2016, pp. 22262230.

[63] Z. Wang, A. C. Bovik, H. R. Sheikh, and E. P. Simoncelli, "Image quality assessment: from error visibility to structural similarity," IEEE transactions on image processing, vol. 13, no. 4, pp. 600-612, 2004.

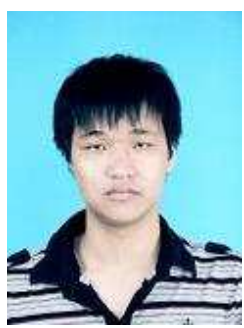

Mingye Ju (SM'16) received a Bachelor degree in communication engineering from Nanjing University of Posts and Telecommunications, Nanjing, China, in 2010 and a Master degree in signal processing from Tianjin University of Technology and Education, Tianjin, China, in 2013. He was with the development department, LongPai vision Company, Ltd., as an Engineer from 2013 to 2014. He is currently a dual-PhD student at Nanjing University of Posts and Telecommunications and University of Technology Sydney. His research interests include computer vision, image processing and machine learning.

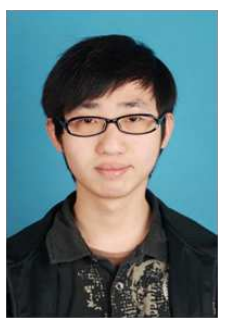

Can Ding (M'16) received a Bachelor degree in Micro-electronics from Xidian University, Xi'an, China, in 2009; and a PHD degreed from Macquarie University, Sydney, Australia, in 2015. From 2012 to 2015 , he is under the Cotutelle agreement between Macquarie University, Australia and Xidian University, China. During this period, he is also with Commonwealth Scientific and Industrial Research Organisation (CSIRO) DPaS Flagship, Marsfield, Australia.

From 2015 to 2017, he was a postdoctoral Research Fellow in University of Technology Sydney (UTS), Sydney, Australia. He is currently a lecturer with Global Big Data Technologies Centre (GBDTC), University of Technology Sydney (UTS), Sydney, Australia. His research interest is in the area of reconfigurable antenna, phase shifter, base station antenna, and $\mathrm{THz}$ waveguides.

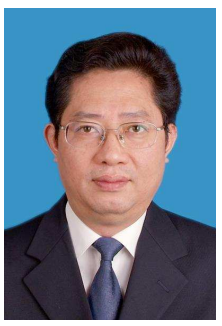

Dengying Zhang (M'17) received the B.S., M.S., and Ph.D. degrees from Nanjing University of Posts and Telecommunication, Nanjing, China, in 1986, 1989, and 2004, respectively. He is currently a Professor of the School of Internet of Things, Nanjing University of Posts and Telecommunication, Nanjing, China. He was a visiting scholar in Digital Media Lab, Umea University, Sweden, from 2007 to 2008. His research interests include signal and information processing, networking technique, and information security. 


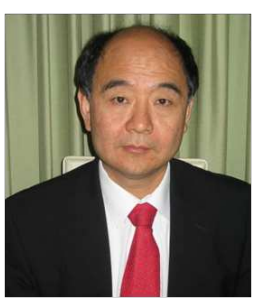

Y. Jay Guo (F'14) received a Bachelor Degree and a Master Degree from Xidian University in 1982 and 1984, respectively, and a $\mathrm{PhD}$ Degree from Xian Jiaotong University in 1987, all in China. He is a Fellow of the Australian Academy of Engineering and Technology, a Fellow of IEEE and a Fellow of IET, and a member of the College of Experts of Australian Research Council (ARC). He has won a number of most prestigious Australian national awards, and was named one of the most influential engineers in Australia in 2014 and 2015. His research interest includes antennas, mm-wave and $\mathrm{THz}$ communications and sensing systems as well as big data. He has published over 400 research papers and holds 22 patents in antennas and wireless systems.

Prof. Guo is a Distinguished Professor and the founding Director of Global Big Data Technologies Centre at the University of Technology Sydney (UTS), Australia. Prior to this appointment in 2014, he served as a Director in CSIRO for over nine years, directing a number of ICT research portfolios. Before joining CSIRO, he held various senior leadership positions in Fujitsu, Siemens and NEC in the U.K.

Prof. Guo has chaired numerous international conferences. He was the International Advisory Committee Chair of IEEE VTC2017, General Chair of ISAP2015, iWAT2014 and WPMC'2014, and TPC Chair of 2010 IEEE WCNC, and 2012 and 2007 IEEE ISCIT. He served as Guest Editor of special issues on "Antennas for Satellite Communications" and "Antennas and Propagation Aspects of 60-90GHz Wireless Communications," both in IEEE Transactions on Antennas and Propagation, Special Issue on "Communications Challenges and Dynamics for Unmanned Autonomous Vehicles," IEEE Journal on Selected Areas in Communications (JSAC), and Special Issue on "5G for Mission Critical Machine Communications", IEEE Network Magazine. 